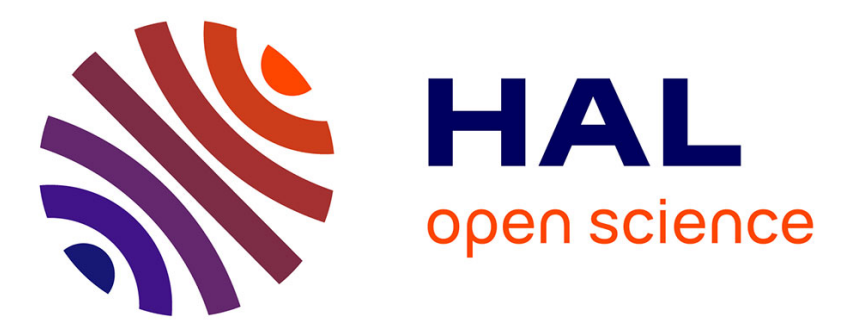

\title{
Relativistic many-body and QED effects on the hyperfine structure of lithium-like ions
}

S. Boucard, P. Indelicato

\section{To cite this version:}

S. Boucard, P. Indelicato. Relativistic many-body and QED effects on the hyperfine structure of lithium-like ions. The European Physical Journal D : Atomic, molecular, optical and plasma physics, 2000, 8 (1), pp.59-73. hal-02093817

\section{HAL Id: hal-02093817 https://hal.sorbonne-universite.fr/hal-02093817}

Submitted on 9 Apr 2019

HAL is a multi-disciplinary open access archive for the deposit and dissemination of scientific research documents, whether they are published or not. The documents may come from teaching and research institutions in France or abroad, or from public or private research centers.
L'archive ouverte pluridisciplinaire HAL, est destinée au dépôt et à la diffusion de documents scientifiques de niveau recherche, publiés ou non, émanant des établissements d'enseignement et de recherche français ou étrangers, des laboratoires publics ou privés. 


\title{
Relativistic Many-Body and QED effects on the hyperfine structure of lithium-like ions
}

\author{
S. Boucard and P. Indelicato \\ Laboratoire Kastler-Brossel Unité Associée No. 18 du CNRS, \\ Ecole Normale Supérieure et Université Pierre et Marie Curie, Case 74, 4 place Jussieu, F-75252 Paris CEDEX 05, France \\ e-mail: paul@spectro.jussieu.fr
}

\begin{abstract}
The hyperfine splitting of the ground state of three-electron ions is studied in detail with the help of the Multi-Configuration Dirac-Fock method. We study the role of the magnetic electron-electron interaction and of the negative energy continuum. An all-order evaluation of some vacuum polarization corrections is performed. The Bohr-Weisskopf effect is evaluated in a simple model.
\end{abstract}

PACS. 32.10.Fn Fine and hyperfine structure-31.30.Jv Relativistic and quantum electrodynamic effects in atoms and molecules - 31.25.Eb Electron correlation calculations for atoms and ions: ground state

\section{Introduction}

With the use of high energy storage rings $[1,2]$ and ElectronBeam Ion traps (super-EBIT) [3], it is now possible to measure the hyperfine splitting of the ground state of heavy ions. Up to now several hydrogen-like ions have been investigated $\left({ }^{185} \mathrm{Re}\right.$ and ${ }^{187} \mathrm{Re}[4],{ }^{165} \mathrm{Ho}[3],{ }^{207} \mathrm{~Pb}$ [2], ${ }^{209} \mathrm{Bi}[1]$ ) by laser or optical spectroscopy. A measurement of the hyperfine splitting of lithium-like Bi has been performed by resolving the two components of the $1 s^{2}$ $2 p_{3 / 2}-1 s^{2} 2 s_{1 / 2} 4 \mathrm{keV} \mathrm{X}$-rays transition. The aim of the study of the hyperfine structure of few-electron heavy ions is to investigate the magnetic sector of strong field QED, while the study of the $1 s$ or $2 s$ Lamb shift in heavy ions probes QED in strong Coulomb fields.

One of the main difficulty in the interpretation of the experiment in term of QED is connected to the knowledge of nuclear effects. The Bohr-Weisskopf [5] effect represents the change in the hyperfine structure energy due to the distribution of magnetic moment of the nucleus, and cannot be evaluated very precisely, due to the lack of knowledge of the nuclear wave function [6-8]. In a recent paper Shabaev et al. $[8,9]$ show that a simultaneous knowledge of the hyperfine splitting of the ground state of one- and three-electron ion with the same $Z$ and $A$ can provide better information to distinguish between the Bohr-Weisskopf and the QED corrections.

As shown in Ref. [4], the study of isotopic series can also help disentangle the two effects. An understanding of the nuclear magnetization distribution is also important for the study of the nuclear anapole moment recently observed in a parity non-conservation experiment [10] or for the search of a proton electric dipole moment (see Ref. [4] and references therein).

In this paper, we calculate many-body effects for the hyperfine splitting of the ground state in the lithium-like sequence by the Multi-Configuration Dirac-Fock method (MCDF). We study the influence of Gaunt correlation and negative energy continuum, following Refs. [11-13]. To be complete, and provide experimentalists with reasonably good splitting energy we include approximate BohrWeisskopf and QED corrections.

The paper is organized as follows. The principle of the calculation is outlined in Sec. 2. We describe the correlated wave function in Sec. 3 and our results are given in Sec. 4. In order to assess the quality of our Bohr-Weisskopf estimates we calculate the hyperfine splitting of the ground state of hydrogen-like ions and compare it with recent experiments. Finally we give our conclusions in Sec. 5.

\section{Principle of the calculation}

\subsection{The MCDF method}

The MCDF method $[14,15]$ is the relativistic equivalent of the Multi-Configuration Hartree-Fock method (MCHF). In order to get a correct relationship between the MCDF method and QED [11,16-18], one has to use the no-pair Hamiltonian

$$
\mathcal{H}^{\text {no pair }}=\sum_{i=1}^{N} \mathcal{H}_{D}\left(r_{i}\right)+\sum_{i<j} \mathcal{V}\left(\left|\mathbf{r}_{i}-\mathbf{r}_{j}\right|\right),
$$

where $\mathcal{H}_{D}$ is the one electron Dirac operator and $\mathcal{V}$ is an operator representing the electron-electron interaction. 
This comes from the one-photon exchange diagram in QED and is gauge dependent. We can use either $\mathcal{V}_{i j}=V_{i j}$ or $\mathcal{V}_{i j}=\Lambda_{i j}^{++} V_{i j} \Lambda_{i j}^{++}$where $\Lambda_{i j}^{++}=\Lambda_{i}^{+} \Lambda_{j}^{+}$is an operator projecting onto the positive energy Dirac eigenstates. The expression of $V_{i j}$ in Coulomb gauge is

$$
\begin{aligned}
V_{i j}= & \frac{1}{r_{i j}}-\frac{\boldsymbol{\alpha}_{i} \cdot \boldsymbol{\alpha}_{j}}{r_{i j}}-\frac{\boldsymbol{\alpha}_{i} \cdot \boldsymbol{\alpha}_{j}}{r_{i j}}\left[\cos \left(\omega_{i j} r_{i j}\right)-1\right] \\
& +\left(\boldsymbol{\alpha}_{i} \cdot \nabla_{i}\right)\left(\boldsymbol{\alpha}_{j} \cdot \nabla_{j}\right) \frac{\cos \left(\omega_{i j} r_{i j}\right)-1}{\omega_{i j}^{2} r_{i j}}
\end{aligned}
$$

where $r_{i j}=\left|\mathbf{r}_{i}-\mathbf{r}_{j}\right|$ is the inter-electronic distance, $\omega_{i j}$ is the energy of the photon exchanged between the two electrons, and $\boldsymbol{\alpha}_{i}$ are the Dirac matrices. The first term represents the Coulomb interaction, the second one is the Gaunt (magnetic) interaction, and the two last terms are the retardation operator. In this expression the $\nabla$ operators do not act on the following wave function but only on the $r_{i j}$.

The atomic wave function is obtained by solving

$$
\mathcal{H}^{n p} \Psi_{\Pi, J, M}\left(\ldots, \mathbf{r}_{i}, \ldots\right)=E_{\Pi, J, M} \Psi_{\Pi, J, M}\left(\ldots, \mathbf{r}_{i}, \ldots\right)
$$

with the aid of a variational principle. $\operatorname{Here} \Psi_{\Pi, J, M}\left(\ldots, \mathbf{r}_{i}, \ldots\right)$ is an eigenfunction of the parity $\Pi$, the total angular momentum $J^{2}$ with eigenvalue $J$ and its projection on the $z$ axis $J_{z}$, with eigenvalue $M$. In the MCDF method, we construct this total wave function as a superposition of configuration state functions (CSF) :

$$
\left|\Psi_{\Pi, J, M}\right\rangle=\sum_{\nu} c_{\nu}|\nu \Pi J M\rangle
$$

The CFS are also eigenfunctions of $\Pi, J^{2}$ and $J_{z}$. The label $\nu$ stands for all other numbers (principal quantum number,...) necessary to define unambiguously the CFS. For a $N$-electron system, the CFS is a linear combination of Slater determinants

$$
|\nu \Pi J M\rangle=\sum_{i} d_{i}\left|\begin{array}{ccc}
\Phi_{1}^{i}\left(r_{1}\right) & \cdots & \Phi_{N}^{i}\left(r_{1}\right) \\
\vdots & \ddots & \vdots \\
\Phi_{1}^{i}\left(r_{N}\right) & \cdots & \Phi_{N}^{i}\left(r_{N}\right)
\end{array}\right|
$$

where the $\Phi$ are the one-electron wave functions. In the relativistic case, they are the Dirac four-component spinors

$$
\Phi_{n \kappa \mu}(\mathbf{r})=\frac{1}{r}\left[\begin{array}{c}
P_{n \kappa}(r) \chi_{\kappa \mu}(\theta, \phi) \\
i Q_{n \kappa}(r) \chi_{-\kappa \mu}(\theta, \phi)
\end{array}\right]
$$

with $\chi_{\kappa \mu}(\theta, \phi)$ the two component Pauli spherical spinors [15] and $P_{n \kappa}(r)$ and $Q_{n \kappa}(r)$ respectively the large and the small radial components of the wave function. The coefficients $d_{i}$ are determined by requiring that each CFS is an eigenstate of $J^{2}$. The functions $P_{n \kappa}(r), Q_{n \kappa}(r)$ are the solutions of coupled integro-differential equations obtained by minimizing Eq. (3) with respect to each radial wave function, while the coefficients $c_{\nu}$ are determined by diagonalization an Hamiltonian matrix (for more details see, e.g., Refs. $[14,15,19])$.

\subsection{Hyperfine structure calculation}

The hyperfine interaction comes from the interaction of the electron and nucleus magnetic moments. The relativistic hyperfine Hamiltonian is given by

$$
H_{h f s}=\sum_{k} \mathrm{M}^{(k)} \cdot \mathrm{T}^{(k)}
$$

where $\mathbf{M}^{(k)}$ and $\mathbf{T}^{(k)}$ are spherical tensor of rank $k$, representing, respectively, the nuclear and the atomic parts of the interaction. We calculate the hyperfine splitting in the ground state which has a total angular momentum $J=1 / 2$. In this case, for angular reasons, only the magnetic dipole term $(k=1)$ is non-zero. Hence the hyperfine matrix element $W\left(J, J^{\prime}\right)$ is written

$$
W\left(J, J^{\prime}\right)=\left\langle I, J, F, M_{F}\left|\mathrm{M}^{(1)} \cdot \mathrm{T}^{(1)}\right| I, J^{\prime}, F, M_{F}\right\rangle,
$$

where $I$ is the nuclear spin and $F$ the total angular momentum of the atom. Equation (8) may be rewritten as (see, e.g., Ref. [20])

$$
\begin{aligned}
W_{J, J^{\prime}}= & (-1)^{I+J+F}\left\{\begin{array}{ccc}
I & J & F \\
J^{\prime} & I & 1
\end{array}\right\} \\
& \times\left\langle I\left\|\mathrm{M}^{(1)}\right\| I\right\rangle\left\langle J\left\|\mathrm{~T}^{(1)}\right\| J^{\prime}\right\rangle .
\end{aligned}
$$

The nuclear magnetic moment $\mu_{I}$ in units of the nuclear magneton $\mu_{N}$ is defined as

$$
\mu_{I} \mu_{N}=\left\langle I I\left|\mathrm{M}_{0}^{(1)}\right| I I\right\rangle=\left(\begin{array}{ccc}
I & 1 & I \\
-I & 0 & I
\end{array}\right)\left\langle I|| \mathrm{M}^{(1)} \| I\right\rangle,
$$

where $\mu_{N}=e \hbar /\left(m_{p} c\right)$ and the $3 j$ symbol is given by

$$
\left(\begin{array}{ccc}
I & 1 & I \\
-I & 0 & I
\end{array}\right)=\sqrt{\frac{I}{(2 I+1)(I+1)}} .
$$

The atomic operator $\mathrm{T}_{q}^{(1)}$ can be expressed as a sum of one-electron operators $\mathrm{t}_{q}^{(1)}[21]$, as

$$
\begin{aligned}
\mathrm{T}_{q}^{(1)} & =\sum \mathrm{t}_{q}^{(1)} \\
& =\sum_{j}-i e \sqrt{\frac{8 \pi}{3}} \frac{\boldsymbol{\alpha}_{j} \cdot \mathrm{Y}_{1 q}^{(0)}\left(\hat{\mathbf{r}}_{j}\right)}{r_{j}^{2}}
\end{aligned}
$$

in which $\boldsymbol{\alpha}$ are the Dirac matrices, $\mathrm{Y}_{1 q}^{(0)}\left(\hat{\mathbf{r}}_{j}\right)$ is a vector spherical harmonic [22] and $j$ represents the $j$ th electron of the atom. Explicit formulas for the matrix elements of $\mathrm{t}_{q}^{(1)}$ are given in Ref. [23]. This operator is only valid for point-like magnetic moment distribution in the nucleus. The radial part of this operator is given by [23]

$\left[r^{k}\right]_{n \kappa n^{\prime} \kappa^{\prime}}=\int_{0}^{\infty} d r r^{k}\left(P_{n \kappa}(r) Q_{n^{\prime} \kappa^{\prime}}(r)+Q_{n \kappa}(r) P_{n^{\prime} \kappa^{\prime}}(r)\right)$.

with $k=-2$. 


\subsection{Nuclear structure corrections}

For heavy elements, a change between a point-like and an extended nuclear charge distribution strongly modifies the wave function near the origin. Since the hyperfine interaction is a short distance interaction, this effect must be accounted for precisely. One nuclear contribution is easily calculated by using a finite charge distribution in the differential equations from which the wave function are deduced. For atomic number larger than 45 we use a Fermi distribution with a thickness parameter $t=2.3 \mathrm{fm}$ and a uniform spherical distribution otherwise. We use the table in Ref. [24], unless more precise nuclear radii are available (see,e.g., [25] and references therein). We also use [25] for deformed nuclei. For isotope not found in Ref. [24] or [25] we modify the nuclear radius using the fitted formula [24]

$$
R^{\prime}-R=0.836\left(A^{\prime 1 / 3}-A^{1 / 3}\right)
$$

where the ' denotes the isotope not found in the table.

In contrast it is very difficult to account for the BohrWeisskopf effect [5] due to the magnetization distribution inside the nucleus. This distribution is not known in general. It depends on the wave function of the unpaired nucleons and on the correlation between nucleons wave functions. Extended calculations have been performed with different models, but agreement between these models is poor $[4,7,6,8]$. As our main purpose here is a study of correlation effects, we use a much simpler model. We suppose that the magnetic moment of the nucleus is spread over a Fermi (or uniform for $Z \leq 45$ ) distribution, with the same mean spherical radius as the charge distribution. This approach gives result in reasonable agreement with more sophisticated models, although it was shown recently on a study of two isotopes of $\mathrm{Rh}$, that the charge and magnetic distribution radii can differ [4].

The formalism we use in this case was derived in Ref. [26] for calculation of the hyperfine anomaly in muonic atoms. In that formalism the Bohr-Weisskopf correction is obtained by replacing the radial part of the matrix element for a point nucleus, defined in Eq. (13) by

$$
\begin{aligned}
{\left[r^{k}\right]_{n \kappa n^{\prime} \kappa^{\prime}}^{\mathrm{B} . \mathrm{W} .} } & \int_{0}^{R_{\mathrm{nuc}}} d s s^{2} \rho_{\mathrm{Mag}}(s) \int_{0}^{s} d r r^{k} \\
& \times\left(P_{n \kappa}(r) Q_{n^{\prime} \kappa^{\prime}}(r)+Q_{n \kappa}(r) P_{n^{\prime} \kappa^{\prime}}(r)\right) .
\end{aligned}
$$

In Eq. (15) $R_{\text {nuc }}$ is the radius of the nuclear distribution, which is infinite in the case of a Fermi distribution, and $\rho_{\text {Mag }}$ is the magnetic moment density,

$$
\rho_{\text {Mag }}(r)=\frac{1}{\mu_{I} \mu_{N}} \frac{m}{1+\exp \frac{r-c}{t}}
$$

where $c$ and $t$ are the Fermi model radius and skin depth and $m$ is defined so that the integral of $\rho_{\text {Mag }}$ over the nuclear volume is equal to $\mu_{I} \mu_{N}$.

\subsection{QED effects}

There has been lately several all-orders calculations of radiative corrections to hyperfine structure of the ground state of 1-electron ions [27-29], and of the ground state of 3-electron ions [8]. These calculations are in first order in $\alpha$ and to all orders in $(Z \alpha)$ and are well adapted for high- $Z$ systems. A complete evaluation of radiative corrections is beyond the scope of the present work. However the effects of the Vacuum polarization in the Uëhling approximation, which comes from changes in the bound-electron wave function, can be relatively easily implemented in the framework of the MCDF method. In practice one only need to add the Uëhling potential to the nuclear potential, to get the contribution of the vacuum polarization to the wave function to all orders. This happens because the MCDF method is based on a direct numerical solution of the wave function differential equation. Many precautions must be taken however to obtain this result as the vacuum polarization potential is singular close to the origin, even when using finite nuclei. The method we used is described in the next subsection.

\subsubsection{Self-consistent Vacuum Polarization}

The logarithmic singularity of the Uëhling potential at the origin for a point charge cannot be easily incorporated in the numerical Dirac solver of our code. We have chosen to limit ourselves to the case of uniform and Fermi charge distributions, for which the singularity is milder. We follow here the formalism of Klarsfeld [30]. For a spherical charge distribution an angular integration of the expression of the Uëhling potential gives [31]

$$
\begin{aligned}
V_{11}(r)= & -\frac{2 \alpha Z \alpha}{3} \frac{1}{r} \int_{0}^{\infty} d r^{\prime} r^{\prime} \rho\left(r^{\prime}\right) \\
& \times\left[\chi_{2}\left(2\left|r-r^{\prime}\right|\right)-\chi_{2}\left(2\left|r+r^{\prime}\right|\right)\right]
\end{aligned}
$$

where the nuclear charge distribution $\rho(r)=\rho_{N} / Z$ is normalized by

$$
4 \pi \int_{0}^{\infty} \rho(r) r^{2} d r=1
$$

The function $\chi_{2}$ belongs to a family of functions defined by

$$
\chi_{n}(x)=\int_{1}^{\infty} e^{-x t} u^{-n}\left(\frac{1}{u}+\frac{1}{2 u^{3}}\right) \sqrt{u^{2}-1} d u .
$$

The expression of the potential at the origin is given by

$$
V_{11}(0)=-\frac{8 \alpha Z \alpha}{3} \int_{0}^{\infty} r^{\prime} \rho\left(r^{\prime}\right) \chi_{1}\left(2 r^{\prime}\right),
$$

while it behaves at large distances as [32]

$$
\begin{gathered}
V_{11}(r)=-\frac{2 \alpha Z \alpha}{3 \pi} \frac{1}{r}\left[\chi_{1}(2 r)+\frac{2}{3}<r^{2}>\chi_{-1}(2 r)\right. \\
\left.+\frac{2}{15}<r^{4}>\chi_{-3}(2 r)+\ldots\right]
\end{gathered}
$$

where

$$
\left\langle r^{2 n}\right\rangle=4 \pi \int_{0}^{\infty} r^{2 n+2} \rho(r) d r
$$


are the moments of the charge distribution.

For a Fermi distribution of parameters $c$ and $t$, we integrate numerically Eqs. (17), (20) and (21). To increase precision the integral is performed with Gauss integrations, and is split into three pieces : from 0 to $c-t$ (GaussLegendre), from $c-t$ to $c+t$ (Gauss-Legendre) and from $c+t$ to $\infty$ (Gauss-Laguerre). This enable to take into account the fast variation of the nuclear charge distribution between $c-t$ and $c+t$.

In the case of an homogeneous distribution of radius $R$, Eq. (17) has been integrated analytically by Klarsfeld [30], with the result

$$
\begin{aligned}
V_{11}(r)=-\frac{\alpha Z \alpha}{2 \pi R^{3}} \frac{1}{r}[ & \frac{2}{5} r+\varphi(2(R+r)) \\
& -\varphi(2(R-r))] \quad(r \leq R) \\
V_{11}(r)=-\frac{\alpha Z \alpha}{2 \pi R^{3}} \frac{1}{r}[ & \varphi(2(r+R))-\varphi(2(r-R)) \\
& \left.+R \chi_{3}(2(r-R))\right] \quad(r>R)
\end{aligned}
$$

where

$$
\varphi(z)=\frac{1}{2} R \chi_{3}(z)+\frac{1}{4} \chi_{4}(z) .
$$

In the same way Eq. (20) gives

$$
V_{11}(0)=-\frac{\alpha Z \alpha}{\pi R^{3}}\left[\frac{1}{5}-R \chi_{2}(2 R)-\frac{1}{2} \chi_{3}(2 R)\right] .
$$

For both distributions the functions $\chi_{n}$ are evaluated numerically by using this development in terms of modified Bessel's functions $K_{0}(z)$ and $K_{1}(z)$, and of the integral Bessel function $K i_{1}(z)$ :

$$
\begin{aligned}
1440 \chi_{4}(z)= & \left(225 z-48 z^{3}-z^{5}\right) K_{0}(z) \\
& -\left(351 z^{2}-46 z^{4}-z^{6}\right) K_{1}(z) \\
& +\left(225+405 z^{2}-45 z^{4}-z^{6}\right) K i_{1}(z), \\
240 \chi_{3}(z)= & \left(33 z^{2}+z^{4}\right) K_{0}(z)+\left(96 z-31 z^{3}-z^{5}\right) K_{1}(z) \\
& -\left(135 z-30 z^{3}-z^{5}\right) K i_{1}(z), \\
48 \chi_{2}(z)= & -\left(21 z+z^{3}\right) K_{0}(z)+\left(19 z+z^{4}\right) K_{1}(z) \\
& +\left(27-18 z^{2}-z^{4}\right) K i_{1}(z), \\
12 \chi_{1}(z)= & \left(12+z^{2}\right) K_{0}(z) \\
& -\left(10 z+z^{3}\right) K_{1}(z)+\left(9 z+z^{3}\right) K i_{1}(z), \\
\chi_{-1}(z)= & \frac{1}{2} K_{0}(z)+\left(\frac{1}{z}-\frac{z}{2}\right) K_{1}(z)+\frac{z}{2} K i_{1}(z), \\
\chi_{-3}(z)= & \frac{3}{z^{2}} K_{0}(z)+\left(\frac{6}{z^{3}}+\frac{3}{2 z}\right) K i_{1}(z) .
\end{aligned}
$$

$K_{0}(z)$ and $K_{1}(z)$ can be evaluated using programs from Ref. [33] and $K i_{1}(z)$ is obtained from

$$
K i_{1}(z)=\int_{z}^{\infty} K_{0}(x) d x=K i_{1}(0)-\int_{0}^{z} K_{0}(x) d x
$$

where $K i_{1}(0)=\pi / 2$ and $[34]$

$$
\begin{aligned}
\int_{0}^{z} K_{0}(x) d x= & -\left(\gamma+\ln \frac{x}{2}\right) x \sum_{k=0}^{\infty} \frac{(x / 2)^{2 k}}{(k !)^{2}(2 k+1)} \\
& +x \sum_{k=0}^{\infty} \frac{(x / 2)^{2 k}}{(k !)^{2}(2 k+1)^{2}} \\
& +x \sum_{k=1}^{\infty} \frac{(x / 2)^{2 k}}{(k !)^{2}(2 k+1)} \\
& \times\left(1+\frac{1}{2}+\ldots+\frac{1}{k}\right)
\end{aligned}
$$

where $\gamma=0.57721 \ldots$ is Euler's constant.

Adding the Uhling potential in the nuclear potential amounts to evaluate the contribution of all diagrams with one or several vacuum polarization loop of the kind displayed on Fig. 1, for both one and two-electron operators. For the exact signification of these diagrams see, e.g., [27, $29,35]$

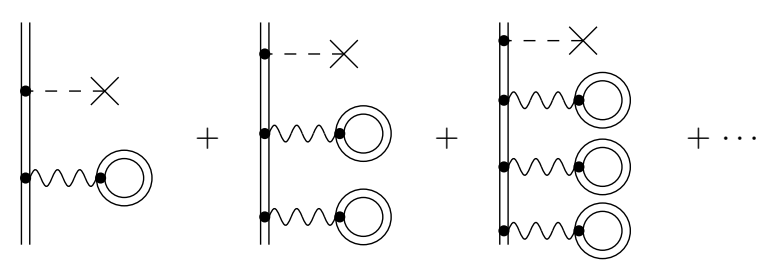

Fig. 1. Feynman diagrams obtained when the Uhling potential is added to the nuclear potential. The dashed lines starting with $\mathrm{a} \times$ represent the hyperfine interaction, the double line a bound electron wavefunction or propagator and the wavy line a retarded photon propagator

\subsubsection{Other QED corrections}

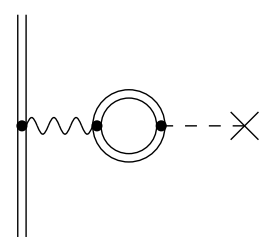

(a)

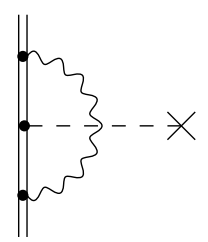

(b)

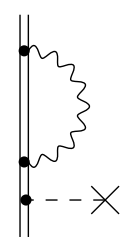

(c)
Fig. 2. Feynman diagrams for other radiative corrections to the HFS energy. See Fig. 1 for the signification of the symbols

We add all other QED corrections of order $\alpha$ (Fig. 2). The first diagram (a) represents a vacuum polarization contribution as given by Schneider et al. [27]. Diagrams (b) and (c) are self-energy corrections. We use the values calculated by Blundell et al. [29]. To get values for arbitrary $Z$ we need to interpolate between Blundell et al. 
values. We make use of the known expansion in $(Z \alpha)$ and $\ln (Z \alpha)^{-2}$ given by [36,37] :

$$
\begin{aligned}
& \Delta E^{\mathrm{SE}}=\frac{\alpha}{\pi} \Delta E^{\mathrm{Hfs}}\left\{\frac{1}{2}+\left(\ln 2-\frac{13}{4}\right) \pi(Z \alpha)\right. \\
& +\left[-\frac{2}{3} \ln ^{2}(Z \alpha)^{-2}\left(\frac{37}{72}-\frac{8}{3} \ln 2\right) \ln (Z \alpha)^{-2}+17.1\right](Z \alpha)^{2} \\
& \left.+\left(\frac{5}{2} \ln 2-\frac{191}{32}\right) \pi(Z \alpha)^{3} \ln (Z \alpha)^{-2}+O\left((Z \alpha)^{3}\right)\right\}
\end{aligned}
$$

where $\Delta E^{\mathrm{Hfs}}$ is the hyperfine energy transition without self-energy correction. To get good agreement at high- $Z$ we then add higher-order terms the coefficients of which are obtained by a linear fit from Blundell's et al. . We obtain :

$$
\begin{aligned}
O\left((Z \alpha)^{3}\right)= & -\left(2.95+0.10925 \ln ^{2}(Z \alpha)^{-2}\right)(Z \alpha)^{3} \\
& -19.798(Z \alpha)^{4}+O\left((Z \alpha)^{4}\right)
\end{aligned}
$$

\section{Evaluation of the correlated wave function}

The MCDF wave function is optimized using the energy as the functional minimized during the self-consistent field (SCF) process (variational process). The most important configurations contributing to the correlation energy of heavy three-electron ions, with spin-orbitals of principal quantum number $n \leq 3$ were determined several years ago [38]. However there is no reason why the most important configurations for the determination of the level energy would be the most important ones for the determination of other operators like the hyperfine structure. We have thus done a complete reanalysis of the configuration set to get the best correlation contribution to the ground state hyperfine energy.

Since we are interested in high- $Z$ ions we had also to include all relevant relativistic effects. In the last few years the importance of including the magnetic interaction in the SCF process, and the role of the negative energy continuum have been studied in detail for two-electron ions [11] and radiative transition probabilities [12]. Recently the conclusions of [12] have been confirmed by an independent Relativistic Many-Body perturbation theory calculation [13]. We have thus done a detailed study of the relative importance of these effects for the Li-like ion ground state hyperfine energy.

Our zeroth-order hyperfine energy is computed with the hyperfine Hamiltonian given by Eq. (7) and a wave function composed of a single configuration $\left(1 s^{2} 2 s\right)$, with only the Coulomb interaction in the SCF process. In the next level of approximation we add the magnetic interaction to SCF process. The effect of correlation is taken into account by adding extra configurations to the ground state one. Configuration added one by one, to find the most important ones until all configuration state functions (CSF) originating from all possible combinations of orbitals of principal quantum number 1 to 3 are included. This gives $25 l s$ configurations or $78 j j$ ones in the case of the ground

\begin{tabular}{cc}
\hline $\begin{array}{c}\text { Configurations } \\
(\mathrm{LS})\end{array}$ & $\begin{array}{c}\text { Number of jj } \\
\text { configurations }\end{array}$ \\
\hline $1 s 2 s 3 s$ & 2 \\
$2 s 3 s^{2}$ & 1 \\
$1 s 3 s^{2}$ & 1 \\
$1 s 2 p 3 p$ & 6 \\
$2 s 2 p^{2}$ & 3 \\
$1 s 3 p^{2}$ & 3 \\
$1 s 2 p^{2}$ & 3 \\
$2 s^{2} 3 s$ & 1 \\
$2 s 3 p^{2}$ & 3 \\
$1 s 3 d^{2}$ & 3 \\
$2 s 3 d^{2}$ & 3 \\
$1 s 2 s^{2}$ & 1 \\
$2 s 2 p 3 p$ & 6 \\
$1 s^{2} 3 s$ & 1 \\
$1 s 2 s 3 d$ & 1 \\
$1 s 3 s 3 d$ & 1 \\
$3 s 3 d^{2}$ & 3 \\
$3 d^{3}$ & 2 \\
$3 p^{2} 3 d$ & 5 \\
$2 s 3 s 3 d$ & 1 \\
$3 s 3 p^{2}$ & 3 \\
$2 p^{2} 3 s$ & 3 \\
$2 p^{2} 3 d$ & 5 \\
$2 p 3 s 3 p$ & 6 \\
$2 p 3 p 3 d$ & 11 \\
Total number (jj) & 78 \\
\hline & \\
\hline
\end{tabular}

Table 1. List of the LS configurations included in the calculation of the correlation energy, by order of importance, with the number of jj configurations they generate.

state $1 s^{2} 2 s$. The list of configurations is presented in Table 1 , in order of the importance of their contribution (for $Z=83$ ). We found that the largest contribution comes from the $1 s 2 s 3 s$ configuration, while this configuration gives an almost negligible contribution to the ground state energy correlation. This is expected since it differs only by one spin-orbital from the main configuration (Brillouin's theorem). The relative contribution of the $1 s 2 s 3 s$ configuration to the total hyperfine correlation energy in the case of a pure Coulomb wave function is presented in Fig. 3. This configuration alone contributes $58 \%$ at $Z=3$ and $98 \%$ at $Z=92$.

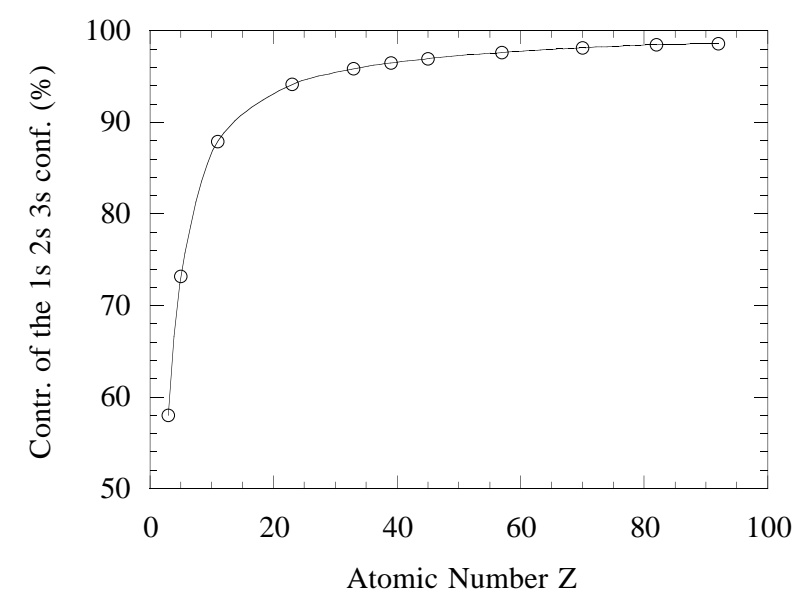

Fig. 3. relative contribution of the $1 \mathrm{~s} 2 \mathrm{~s} 3 \mathrm{~s}$ configuration to the total hyperfine correlation energy in the case of a pure Coulomb wave function. 


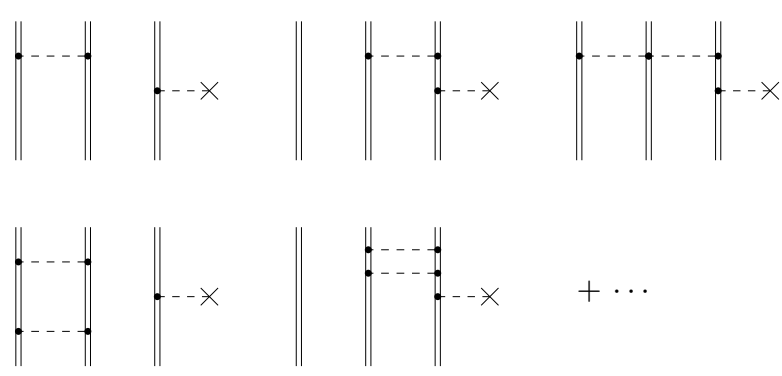

(a)

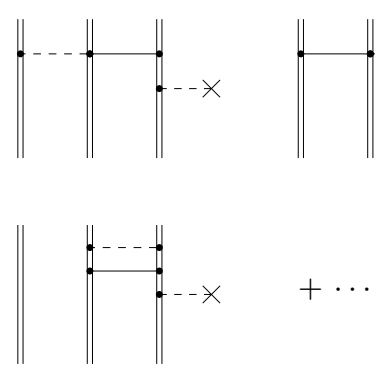

(b)

Fig. 4. Feynman diagrams for the ladder approximation : (a) Magnetic interaction in the SCF process, (b) CoulombMagnetic mixed terms.

There are other differences with respect to the evaluation of the level energy. When computing energy to an approximation beyond first order, care must be taken to exclude the negative continuum, in order to really be in the no-pair approximation $[17,25]$. For one-electron operators like the transition rate or hyperfine splitting it has been recently shown that the correlation contribution must be evaluated with a wave function which include contributions from the negative energy continuum $[12,13,39]$, in order to get high accuracy. We have thus evaluated the ground state hyperfine energy with wave functions evaluated with or without projection operators. At high- $Z$, calculating the MCDF wave function without projection operators induces severe convergence problems during the variational process where the energy is minimized. We thus have to reduce the number of correlation configurations for $Z \geq 45$. We conserve only configurations containing orbitals $1 s, 2 s, 2 p$ and $3 s$. This is not however a very strong limitation, due to the dominance of the $1 s 2 s 3 s$ configuration in the correlation.

Up to this point, we have treated the Gaunt interaction and the retardation term in (2) as perturbations. For the retardation we must continue to do so because $\omega_{i j}$ can be defined only for occupied orbitals, i.e., $1 s$ and $2 s$, for which the diagonal Lagrange parameter in the DiracFock equation, used to insure normalisation, can be interpreted as an approximate binding energy, due to Koopmans' theorem (see Ref. [11]). However we can include the Gaunt term in the self-consistent process. This gives two kinds of extra contributions. The first kind amounts to include more and more of the magnetic ladder diagrams (Fig. 4(a)), and shows up mostly as the contribution of ex-

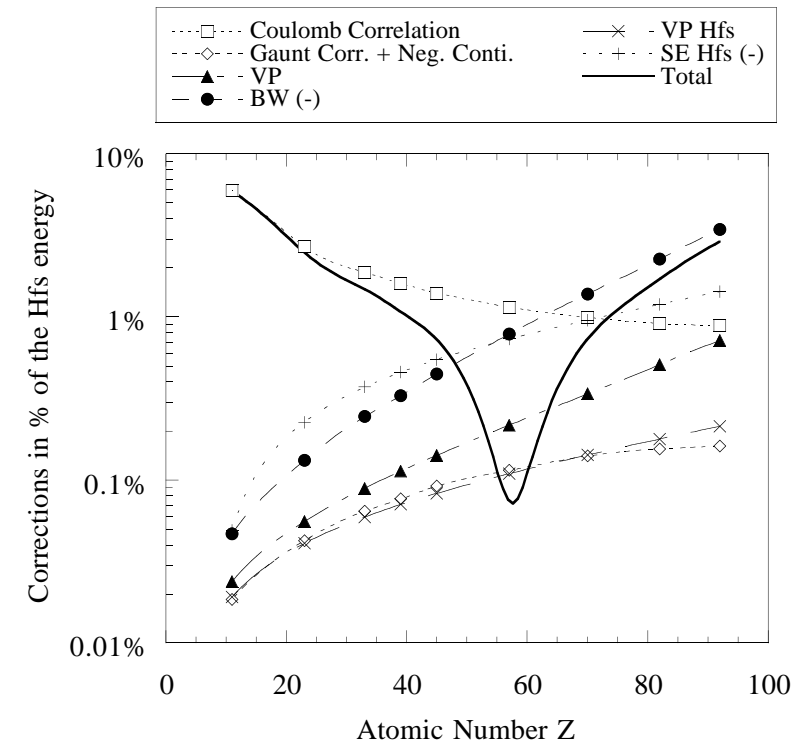

Fig. 5. Summary of all contributions presented in the present work. Absolute values of the ratio of each contribution to the total HFS energy is plotted. Correlation: Coulomb correlation. Gaunt Corr. + Neg. Conti.: Sum of the Gaunt correlation and Negative continuum (Coulomb and Gaunt part) contributions. VP: Effect of self-consistent vacuum polarization. BW: Bohr-Weisskopf effect. VP Hfs, SE Hfs: radiative corrections (Vacuum polarization and Self-energy to the hyperfine interaction. Total: Sum of all correlation. The quink in the Total curve corresponds to a change of sign.

tra magnetic terms in the Hamiltonian, while the second represents Coulomb-magnetic mixed diagrams (Fig. 4(b)) and shows up through changes of the radial wave functions due to magnetic contributions to the Dirac-Fock differential equations.

\section{RESULTS AND DISCUSSION}

\section{1 atomic effects}

The total ground state $1 s^{2} 2 s$ hyperfine transition energy for lithium-like ions $3 \leq Z \leq 92$ (stable isotopes) are presented in Table 2, with all corrections discussed in the preceding sections. The nuclear magnetic moments come from Raghavan's table [40] and from Refs. [41,42] in the case of ${ }^{165} \mathrm{Ho}^{66+}$. We have listed separately the hyperfine transition $\Delta E_{L i}^{H f s}$ and the Bohr-Weisskopf correction $\Delta E_{L i}^{B W} . \Delta E_{L i}^{H f s}$ includes correlation (Coulomb and magnetic) and the self-consistent vacuum polarization (see Sec. 2.4.1). $V P_{L i}^{h f s}$ and $S E_{L i}$ are the QED corrections given by Refs. [27] and [29] respectively (see Sec. 2.4.2). The total transition energies $\Delta E_{L i}$ are expressed in $\mathrm{eV}$ and the wavelengths $\lambda_{L i}$ in $\mathrm{nm}$.

We can follow the evolution of all the corrections as a function of the atomic number $Z$ (Fig. 5). The sum of all corrections computed in the present paper increases the hyperfine transition energy by about $6 \%$ at $Z=11$ but 


\begin{tabular}{|c|c|c|c|c|c|c|c|c|}
\hline Label & $I$ & $\mu_{I}$ & $\Delta E_{L i}^{H f s}$ & $\Delta E_{L i}^{B W}$ & $V P_{L i}^{h f s}$ & $S E_{L i}$ & $\Delta E_{L i}$ & $\lambda_{L i}$ \\
\hline${ }^{6} \mathrm{Li}$ & 1 & 0.8220473 & $9.1745 \times 10^{-07}$ & $-8.6713 \times 10^{-11}$ & $3.8675 \times 10^{-11}$ & $4.8535 \times 10^{-10}$ & $9.1789 \times 10^{-07}$ & $1.3508 \times 10^{+09}$ \\
\hline${ }^{7} \mathrm{Li}$ & $3 / 2$ & 3.2564268 & $3.2306 \times 10^{-06}$ & $-3.1694 \times 10^{-10}$ & $1.3618 \times 10^{-10}$ & $1.7091 \times 10^{-09}$ & $3.2321 \times 10^{-06}$ & $3.8360 \times 10^{+08}$ \\
\hline${ }^{9} \mathrm{Be}^{1+}$ & $3 / 2$ & -1.177492 & $5.1603 \times 10^{-06}$ & $-7.3064 \times 10^{-10}$ & $3.1775 \times 10^{-10}$ & $2.3523 \times 10^{-09}$ & $5.1623 \times 10^{-06}$ & $2.4017 \times 10^{+08}$ \\
\hline${ }^{10} \mathrm{~B}^{2+}$ & 3 & 1.800645 & $1.7604 \times 10^{-05}$ & $-2.9010 \times 10^{-09}$ & $1.4136 \times 10^{-09}$ & $6.0722 \times 10^{-09}$ & $1.7608 \times 10^{-05}$ & $7.0413 \times 10^{+07}$ \\
\hline${ }^{11} \mathrm{~B}^{2+}$ & $3 / 2$ & 2.688649 & $3.0040 \times 10^{-05}$ & $-5.0747 \times 10^{-09}$ & $2.4122 \times 10^{-09}$ & $1.0362 \times 10^{-08}$ & $3.0048 \times 10^{-05}$ & $4.1263 \times 10^{+07}$ \\
\hline${ }^{13} \mathrm{C}^{3+}$ & $1 / 2$ & 0.702412 & $2.3500 \times 10^{-05}$ & $-4.9809 \times 10^{-09}$ & $2.3343 \times 10^{-09}$ & $5.1640 \times 10^{-09}$ & $2.3503 \times 10^{-05}$ & $5.2753 \times 10^{+07}$ \\
\hline${ }^{14} \mathrm{~N}^{4+}$ & 1 & 0.403761 & $1.7668 \times 10^{-05}$ & $-4.4337 \times 10^{-09}$ & $1.9517 \times 10^{-09}$ & $1.4962 \times 10^{-09}$ & $1.7667 \times 10^{-05}$ & $7.0179 \times 10^{+07}$ \\
\hline${ }^{15} \mathrm{~N}^{4+}$ & $1 / 2$ & -0.283189 & $1.6522 \times 10^{-05}$ & $-4.2312 \times 10^{-09}$ & $1.4459 \times 10^{-09}$ & $1.3992 \times 10^{-09}$ & $1.6521 \times 10^{-05}$ & $7.5045 \times 10^{+07}$ \\
\hline${ }^{17} \mathrm{O}^{5+}$ & $5 / 2$ & -1.89379 & $1.0574 \times 10^{-04}$ & $-3.2902 \times 10^{-08}$ & $1.4459 \times 10^{-08}$ & $-5.9160 \times 10^{-09}$ & $1.0571 \times 10^{-04}$ & $1.1728 \times 10^{+07}$ \\
\hline${ }^{19} \mathrm{~F}^{6+}$ & $1 / 2$ & 2.628868 & $3.6608 \times 10^{-04}$ & $-1.3528 \times 10^{-07}$ & $5.6820 \times 10^{-08}$ & $-7.3172 \times 10^{-08}$ & $3.6593 \times 10^{-04}$ & $3.3882 \times 10^{+06}$ \\
\hline${ }^{21} \mathrm{Ne}^{7+}$ & $3 / 2$ & -0.661797 & $8.7631 \times 10^{-05}$ & $-3.8071 \times 10^{-08}$ & $1.5209 \times 10^{-08}$ & $-3.0302 \times 10^{-08}$ & $8.7578 \times 10^{-05}$ & $1.4157 \times 10^{+07}$ \\
\hline${ }^{23} \mathrm{Na}^{8+}$ & $3 / 2$ & 2.217656 & $4.0346 \times 10^{-04}$ & $-1.8906 \times 10^{-07}$ & $7.7388 \times 10^{-08}$ & $-1.9885 \times 10^{-07}$ & $4.0315 \times 10^{-04}$ & $3.0754 \times 10^{+06}$ \\
\hline${ }^{25} \mathrm{Mg}^{9+}$ & $5 / 2$ & -0.85545 & $1.8672 \times 10^{-04}$ & $-9.9180 \times 10^{-08}$ & $3.9208 \times 10^{-08}$ & $-1.1962 \times 10^{-07}$ & $1.8654 \times 10^{-04}$ & $6.6464 \times 10^{+06}$ \\
\hline${ }^{27} \mathrm{Al}^{10+}$ & $5 / 2$ & 3.641507 & $1.0338 \times 10^{-03}$ & $-5.9006 \times 10^{-07}$ & $2.3579 \times 10^{-07}$ & $-8.1533 \times 10^{-07}$ & $1.0326 \times 10^{-03}$ & $1.2007 \times 10^{+06}$ \\
\hline${ }^{29} \mathrm{Si}^{11+}$ & $1 / 2$ & -0.55529 & $3.3469 \times 10^{-04}$ & $-2.1368 \times 10^{-07}$ & $8.2376 \times 10^{-08}$ & $-3.1355 \times 10^{-07}$ & $3.3424 \times 10^{-04}$ & $3.7094 \times 10^{+06}$ \\
\hline${ }^{31} \mathrm{P}^{12+}$ & $1 / 2$ & 1.1316 & $8.5374 \times 10^{-04}$ & $-5.9983 \times 10^{-07}$ & $2.2550 \times 10^{-07}$ & $-9.2625 \times 10^{-07}$ & $8.5244 \times 10^{-04}$ & $1.4545 \times 10^{+06}$ \\
\hline${ }^{33} \mathrm{~S}^{13+}$ & $3 / 2$ & 0.643821 & $3.9927 \times 10^{-04}$ & $-3.0898 \times 10^{-07}$ & $1.1263 \times 10^{-07}$ & $-4.9223 \times 10^{-07}$ & $3.9858 \times 10^{-04}$ & $3.1106 \times 10^{+06}$ \\
\hline${ }^{35} \mathrm{Cl}^{14+}$ & $3 / 2$ & 0.821874 & $6.2028 \times 10^{-04}$ & $-5.2402 \times 10^{-07}$ & $1.8610 \times 10^{-07}$ & $-8.5628 \times 10^{-07}$ & $6.1909 \times 10^{-04}$ & $2.0027 \times 10^{+06}$ \\
\hline${ }^{37} \mathrm{Cl}^{14+}$ & $3 / 2$ & 0.684124 & $5.1630 \times 10^{-04}$ & $-4.4275 \times 10^{-07}$ & $1.5490 \times 10^{-07}$ & $-7.1274 \times 10^{-07}$ & $5.1530 \times 10^{-04}$ & $2.4060 \times 10^{+06}$ \\
\hline${ }^{39} \mathrm{~K}^{16+}$ & $3 / 2$ & 0.391507 & $4.2339 \times 10^{-04}$ & $-4.1982 \times 10^{-07}$ & $1.4220 \times 10^{-07}$ & $-7.0910 \times 10^{-07}$ & $4.2241 \times 10^{-04}$ & $2.9352 \times 10^{+06}$ \\
\hline${ }^{41} \mathrm{~K}^{16+}$ & $3 / 2$ & 0.214893 & $2.3239 \times 10^{-04}$ & $-2.3356 \times 10^{-07}$ & $7.8049 \times 10^{-08}$ & $-3.8920 \times 10^{-07}$ & $2.3184 \times 10^{-04}$ & $5.3478 \times 10^{+06}$ \\
\hline${ }^{43} \mathrm{Ca}^{17+}$ & $7 / 2$ & -1.317643 & $1.4416 \times 10^{-03}$ & $-1.5704 \times 10^{-06}$ & $5.0998 \times 10^{-07}$ & $-2.6258 \times 10^{-06}$ & $1.4379 \times 10^{-03}$ & $8.6227 \times 10^{+05}$ \\
\hline${ }^{45} \mathrm{Sc}^{18+}$ & $7 / 2$ & 4.756487 & $6.0934 \times 10^{-03}$ & $-7.0247 \times 10^{-06}$ & $2.2648 \times 10^{-06}$ & $-1.1990 \times 10^{-05}$ & $6.0767 \times 10^{-03}$ & $2.0403 \times 10^{+05}$ \\
\hline${ }^{47} \mathrm{Ti}^{19+}$ & $5 / 2$ & -0.78848 & $1.2329 \times 10^{-03}$ & $-1.5204 \times 10^{-06}$ & $4.8037 \times 10^{-07}$ & $-2.6060 \times 10^{-06}$ & $1.2293 \times 10^{-03}$ & $1.0086 \times 10^{+06}$ \\
\hline${ }^{49} \mathrm{Ti}^{19+}$ & $7 / 2$ & -1.10417 & $1.6443 \times 10^{-03}$ & $-2.0510 \times 10^{-06}$ & $6.4064 \times 10^{-07}$ & $-3.4754 \times 10^{-06}$ & $1.6394 \times 10^{-03}$ & $7.5629 \times 10^{+05}$ \\
\hline${ }^{51} \mathrm{~V}^{20+}$ & $7 / 2$ & 5.148706 & $8.8551 \times 10^{-03}$ & $-1.1624 \times 10^{-05}$ & $3.6092 \times 10^{-06}$ & $-2.0006 \times 10^{-05}$ & $8.8271 \times 10^{-03}$ & $1.4046 \times 10^{+05}$ \\
\hline${ }^{53} \mathrm{Cr}^{21+}$ & $3 / 2$ & -0.47454 & $1.0931 \times 10^{-03}$ & $-1.5448 \times 10^{-06}$ & $4.6519 \times 10^{-07}$ & $-2.6284 \times 10^{-06}$ & $1.0894 \times 10^{-03}$ & $1.1381 \times 10^{+06}$ \\
\hline${ }^{55} \mathrm{Mn}^{22+}$ & $5 / 2$ & 3.468719 & $8.2115 \times 10^{-03}$ & $-1.2333 \times 10^{-05}$ & $3.6426 \times 10^{-06}$ & $-2.0935 \times 10^{-05}$ & $8.1819 \times 10^{-03}$ & $1.5154 \times 10^{+05}$ \\
\hline${ }^{57} \mathrm{Fe}^{23+}$ & $1 / 2$ & 0.090623 & $4.0626 \times 10^{-04}$ & $-6.5094 \times 10^{-07}$ & $1.8756 \times 10^{-07}$ & $-1.0945 \times 10^{-06}$ & $4.0470 \times 10^{-04}$ & $3.0636 \times 10^{+06}$ \\
\hline${ }^{59} \mathrm{Co}^{24+}$ & $7 / 2$ & 4.627 & $1.3407 \times 10^{-02}$ & $-2.2754 \times 10^{-05}$ & $6.4326 \times 10^{-06}$ & $-3.8055 \times 10^{-05}$ & $1.3353 \times 10^{-02}$ & $9.2853 \times 10^{+04}$ \\
\hline${ }^{61} \mathrm{Ni}^{25+}$ & $3 / 2$ & -0.75002 & $2.8558 \times 10^{-03}$ & $-5.1714 \times 10^{-06}$ & $1.4221 \times 10^{-06}$ & $-8.5177 \times 10^{-06}$ & $2.8436 \times 10^{-03}$ & $4.3602 \times 10^{+05}$ \\
\hline${ }^{63} \mathrm{Cu}^{26+}$ & $3 / 2$ & 2.227346 & $9.5160 \times 10^{-03}$ & $-1.8338 \times 10^{-05}$ & $4.9125 \times 10^{-06}$ & $-2.9751 \times 10^{-05}$ & $9.4728 \times 10^{-03}$ & $1.3088 \times 10^{+05}$ \\
\hline${ }^{65} \mathrm{Cu}^{26+}$ & $3 / 2$ & 2.38167 & $1.0175 \times 10^{-02}$ & $-1.9774 \times 10^{-05}$ & $5.2526 \times 10^{-06}$ & $-3.1811 \times 10^{-05}$ & $1.0129 \times 10^{-02}$ & $1.2241 \times 10^{+05}$ \\
\hline${ }^{67} \mathrm{Zn}^{27+}$ & $5 / 2$ & 0.875479 & $3.7634 \times 10^{-03}$ & $-7.8078 \times 10^{-06}$ & $2.0118 \times 10^{-06}$ & $-1.2307 \times 10^{-05}$ & $3.7453 \times 10^{-03}$ & $3.3104 \times 10^{+05}$ \\
\hline${ }^{69} \mathrm{Ga}^{28+}$ & $3 / 2$ & 2.016589 & $1.0734 \times 10^{-02}$ & $-2.3295 \times 10^{-05}$ & $5.9358 \times 10^{-06}$ & $-3.6645 \times 10^{-05}$ & $1.0680 \times 10^{-02}$ & $1.1608 \times 10^{+05}$ \\
\hline${ }^{71} \mathrm{Ga}^{28+}$ & $3 / 2$ & 2.562266 & $1.3638 \times 10^{-02}$ & $-2.9826 \times 10^{-05}$ & $7.5416 \times 10^{-06}$ & $-4.6559 \times 10^{-05}$ & $1.3570 \times 10^{-02}$ & $9.1369 \times 10^{+04}$ \\
\hline${ }^{73} \mathrm{Ge}^{29+}$ & $9 / 2$ & -0.879468 & $4.3339 \times 10^{-03}$ & $-1.0038 \times 10^{-05}$ & $2.4766 \times 10^{-06}$ & $-1.5417 \times 10^{-05}$ & $4.3109 \times 10^{-03}$ & $2.8760 \times 10^{+05}$ \\
\hline${ }^{75} \mathrm{As}^{30+}$ & $3 / 2$ & 1.439475 & $9.4302 \times 10^{-03}$ & $-2.2994 \times 10^{-05}$ & $5.5643 \times 10^{-06}$ & $-3.4898 \times 10^{-05}$ & $9.3779 \times 10^{-03}$ & $1.3221 \times 10^{+05}$ \\
\hline${ }^{77} \mathrm{Se}^{31+}$ & $1 / 2$ & 0.535042 & $5.8093 \times 10^{-03}$ & $-1.4890 \times 10^{-05}$ & $3.5363 \times 10^{-06}$ & $-2.2331 \times 10^{-05}$ & $5.7756 \times 10^{-03}$ & $2.1467 \times 10^{+05}$ \\
\hline${ }^{79} \mathrm{Br}^{32+}$ & $3 / 2$ & 2.1064 & $1.6805 \times 10^{-02}$ & $-4.5342 \times 10^{-05}$ & $1.0545 \times 10^{-05}$ & $-6.7004 \times 10^{-05}$ & $1.6703 \times 10^{-02}$ & $7.4230 \times 10^{+04}$ \\
\hline${ }^{81} \mathrm{Br}^{32+}$ & $3 / 2$ & 2.270562 & $1.8113 \times 10^{-02}$ & $-4.9202 \times 10^{-05}$ & $1.1367 \times 10^{-05}$ & $-7.2222 \times 10^{-05}$ & $1.8003 \times 10^{-02}$ & $6.8868 \times 10^{+04}$ \\
\hline${ }^{83} \mathrm{Kr}^{33+}$ & $9 / 2$ & -0.970669 & $7.0961 \times 10^{-03}$ & $-2.0103 \times 10^{-05}$ & $4.5872 \times 10^{-06}$ & $-2.9311 \times 10^{-05}$ & $7.0512 \times 10^{-03}$ & $1.7583 \times 10^{+05}$ \\
\hline${ }^{85} \mathrm{Rb}^{34+}$ & $5 / 2$ & 1.357 & $1.1755 \times 10^{-02}$ & $-3.5304 \times 10^{-05}$ & $7.8223 \times 10^{-06}$ & $-5.0240 \times 10^{-05}$ & $1.1677 \times 10^{-02}$ & $1.0618 \times 10^{+05}$ \\
\hline${ }^{87} \mathrm{Sr}^{35+}$ & $9 / 2$ & -1.093603 & $9.6062 \times 10^{-03}$ & $-2.9759 \times 10^{-05}$ & $6.5765 \times 10^{-06}$ & $-4.2436 \times 10^{-05}$ & $9.5406 \times 10^{-03}$ & $1.2995 \times 10^{+05}$ \\
\hline${ }^{89} \mathrm{Y}^{36+}$ & $1 / 2$ & -0.137415 & $2.3744 \times 10^{-03}$ & $-7.7686 \times 10^{-06}$ & $1.6713 \times 10^{-06}$ & $-1.0830 \times 10^{-05}$ & $2.3575 \times 10^{-03}$ & $5.2591 \times 10^{+05}$ \\
\hline${ }^{91} \mathrm{Zr}^{37+}$ & $5 / 2$ & -1.30362 & $1.4744 \times 10^{-02}$ & $-5.0795 \times 10^{-05}$ & $1.0664 \times 10^{-05}$ & $-6.9371 \times 10^{-05}$ & $1.4635 \times 10^{-02}$ & $8.4720 \times 10^{+04}$ \\
\hline${ }^{93} \mathrm{Nb}^{38+}$ & $9 / 2$ & 6.1705 & $7.0377 \times 10^{-02}$ & $-2.5452 \times 10^{-04}$ & $5.2281 \times 10^{-05}$ & $-3.4126 \times 10^{-04}$ & $6.9834 \times 10^{-02}$ & $1.7754 \times 10^{+04}$ \\
\hline${ }^{95} \mathrm{Mo}^{39+}$ & $5 / 2$ & -0.9142 & $1.2245 \times 10^{-02}$ & $-4.6578 \times 10^{-05}$ & $9.3374 \times 10^{-06}$ & $-6.1142 \times 10^{-05}$ & $1.2146 \times 10^{-02}$ & $1.0208 \times 10^{+05}$ \\
\hline${ }^{97} \mathrm{Mo}^{39+}$ & $5 / 2$ & -0.9335 & $1.2502 \times 10^{-02}$ & $-4.7820 \times 10^{-05}$ & $9.5339 \times 10^{-06}$ & $-6.2428 \times 10^{-05}$ & $1.2402 \times 10^{-02}$ & $9.9975 \times 10^{+04}$ \\
\hline${ }^{99} \mathrm{Tc}^{40+}$ & $9 / 2$ & 5.6847 & $7.6536 \times 10^{-02}$ & $-3.0820 \times 10^{-04}$ & $5.9884 \times 10^{-05}$ & $-3.9324 \times 10^{-04}$ & $7.5895 \times 10^{-02}$ & $1.6336 \times 10^{+04}$ \\
\hline${ }^{101} \mathrm{Ru}^{41+}$ & $5 / 2$ & -0.7188 & $1.1332 \times 10^{-02}$ & $-4.7805 \times 10^{-05}$ & $9.0936 \times 10^{-06}$ & $-5.9867 \times 10^{-05}$ & $1.1234 \times 10^{-02}$ & $1.1037 \times 10^{+05}$ \\
\hline${ }^{99} \mathrm{Ru}^{41+}$ & $5 / 2$ & -0.6413 & $1.0111 \times 10^{-02}$ & $-4.2433 \times 10^{-05}$ & $8.1137 \times 10^{-06}$ & $-5.3416 \times 10^{-05}$ & $1.0023 \times 10^{-02}$ & $1.2369 \times 10^{+05}$ \\
\hline${ }^{103} \mathrm{Rh}^{42+}$ & $1 / 2$ & -0.0884 & $2.5150 \times 10^{-03}$ & $-1.1138 \times 10^{-05}$ & $2.0689 \times 10^{-06}$ & $-1.3652 \times 10^{-05}$ & $2.4923 \times 10^{-03}$ & $4.9747 \times 10^{+05}$ \\
\hline${ }^{105} \mathrm{Pd}^{43+}$ & $5 / 2$ & -0.642 & $1.1855 \times 10^{-02}$ & $-5.5361 \times 10^{-05}$ & $9.9943 \times 10^{-06}$ & $-6.6085 \times 10^{-05}$ & $1.1743 \times 10^{-02}$ & $1.0558 \times 10^{+05}$ \\
\hline${ }^{107} \mathrm{Ag}^{44+}$ & $1 / 2$ & -0.11368 & $3.7786 \times 10^{-03}$ & $-1.8615 \times 10^{-05}$ & $3.2632 \times 10^{-06}$ & $-2.1617 \times 10^{-05}$ & $3.7417 \times 10^{-03}$ & $3.3136 \times 10^{+05}$ \\
\hline${ }^{109} \mathrm{Ag}^{44+}$ & $1 / 2$ & -0.130691 & $4.3437 \times 10^{-03}$ & $-2.1497 \times 10^{-05}$ & $3.7512 \times 10^{-06}$ & $-2.4849 \times 10^{-05}$ & $4.3011 \times 10^{-03}$ & $2.8826 \times 10^{+05}$ \\
\hline${ }^{111} \mathrm{Cd}^{45+}$ & $1 / 2$ & -0.594886 & $2.1334 \times 10^{-02}$ & $-1.1031 \times 10^{-04}$ & $1.8866 \times 10^{-05}$ & $-1.2518 \times 10^{-04}$ & $2.1118 \times 10^{-02}$ & $5.8711 \times 10^{+04}$ \\
\hline${ }^{113} \mathrm{Cd}^{45+}$ & $1 / 2$ & -0.622301 & $2.2316 \times 10^{-02}$ & $-1.1589 \times 10^{-04}$ & $1.9734 \times 10^{-05}$ & $-1.3094 \times 10^{-04}$ & $2.2088 \times 10^{-02}$ & $5.6131 \times 10^{+04}$ \\
\hline
\end{tabular}

Table 2. Hyperfine transition energy $\Delta E_{L i}$ in $[\mathrm{eV}]$ and in wavelength $\lambda_{L i}[\mathrm{~nm}]$. 


\begin{tabular}{|c|c|c|c|c|c|c|c|c|}
\hline Label & $I$ & $\mu_{I}$ & $\Delta E_{L i}^{H f s}$ & $\Delta E_{L i}^{B W}$ & $V P_{L i}^{h f s}$ & $S E_{L i}$ & $\Delta E_{L i}$ & $\lambda_{L i}$ \\
\hline${ }^{113} \operatorname{In}^{46+}$ & $9 / 2$ & 5.5289 & $1.1873 \times 10^{-01}$ & $-6.4175 \times 10^{-04}$ & $1.0748 \times 10^{-04}$ & $-7.1417 \times 10^{-04}$ & $1.1749 \times 10^{-01}$ & $1.0553 \times 10^{+04}$ \\
\hline${ }^{115} \operatorname{In}^{46+}$ & $9 / 2$ & 5.5408 & $1.1898 \times 10^{-01}$ & $-6.4585 \times 10^{-04}$ & $1.0770 \times 10^{-04}$ & $-7.1565 \times 10^{-04}$ & $1.1773 \times 10^{-01}$ & $1.0532 \times 10^{+04}$ \\
\hline${ }^{115} \mathrm{Sn}^{47+}$ & $1 / 2$ & -0.91883 & $3.8252 \times 10^{-02}$ & $-2.1446 \times 10^{-04}$ & $3.5432 \times 10^{-05}$ & $-2.3574 \times 10^{-04}$ & $3.7837 \times 10^{-02}$ & $3.2768 \times 10^{+04}$ \\
\hline${ }^{117} \mathrm{Sn}^{47+}$ & $1 / 2$ & -1.00104 & $4.1670 \times 10^{-02}$ & $-2.3462 \times 10^{-04}$ & $3.8598 \times 10^{-05}$ & $-2.5681 \times 10^{-04}$ & $4.1218 \times 10^{-02}$ & $3.0080 \times 10^{+04}$ \\
\hline${ }^{119} \mathrm{Sn}^{47+}$ & $1 / 2$ & -1.04728 & $4.3592 \times 10^{-02}$ & $-2.4646 \times 10^{-04}$ & $4.0378 \times 10^{-05}$ & $-2.6865 \times 10^{-04}$ & $4.3117 \times 10^{-02}$ & $2.8755 \times 10^{+04}$ \\
\hline${ }^{121} \mathrm{Sb}^{48+}$ & $5 / 2$ & 3.3634 & $9.0352 \times 10^{-02}$ & $-5.3753 \times 10^{-04}$ & $8.5614 \times 10^{-05}$ & $-5.7025 \times 10^{-04}$ & $8.9330 \times 10^{-02}$ & $1.3879 \times 10^{+04}$ \\
\hline${ }^{123} \mathrm{Sb}^{48+}$ & $7 / 2$ & 2.5498 & $6.5228 \times 10^{-02}$ & $-3.8961 \times 10^{-04}$ & $6.1808 \times 10^{-05}$ & $-4.1169 \times 10^{-04}$ & $6.4489 \times 10^{-02}$ & $1.9226 \times 10^{+04}$ \\
\hline${ }^{123} \mathrm{Te}^{49+}$ & $1 / 2$ & -0.736948 & $3.5465 \times 10^{-02}$ & $-2.2037 \times 10^{-04}$ & $3.4367 \times 10^{-05}$ & $-2.2913 \times 10^{-04}$ & $3.5050 \times 10^{-02}$ & $3.5374 \times 10^{+04}$ \\
\hline${ }^{125} \mathrm{Te}^{49+}$ & $1 / 2$ & -0.888505 & $4.2754 \times 10^{-02}$ & $-2.6670 \times 10^{-04}$ & $4.1431 \times 10^{-05}$ & $-2.7623 \times 10^{-04}$ & $4.2253 \times 10^{-02}$ & $2.9343 \times 10^{+04}$ \\
\hline${ }^{127} \mathrm{I}^{50+}$ & $5 / 2$ & 2.813273 & $8.7239 \times 10^{-02}$ & $-5.6634 \times 10^{-04}$ & $8.6431 \times 10^{-05}$ & $-5.7673 \times 10^{-04}$ & $8.6182 \times 10^{-02}$ & $1.4386 \times 10^{+04}$ \\
\hline${ }^{129} \mathrm{Xe}^{51+}$ & $1 / 2$ & -0.777976 & $4.3154 \times 10^{-02}$ & $-2.9125 \times 10^{-04}$ & $4.3699 \times 10^{-05}$ & $-2.9180 \times 10^{-04}$ & $4.2614 \times 10^{-02}$ & $2.9094 \times 10^{+04}$ \\
\hline${ }^{131} \mathrm{Xe}^{51+}$ & $3 / 2$ & 0.691862 & $2.5582 \times 10^{-02}$ & $-1.7330 \times 10^{-04}$ & $2.5906 \times 10^{-05}$ & $-1.7298 \times 10^{-04}$ & $2.5262 \times 10^{-02}$ & $4.9080 \times 10^{+04}$ \\
\hline${ }^{133} \mathrm{Cs}^{52+}$ & $7 / 2$ & 2.582025 & $8.7752 \times 10^{-02}$ & $-6.2120 \times 10^{-04}$ & $9.0802 \times 10^{-05}$ & $-6.0669 \times 10^{-04}$ & $8.6615 \times 10^{-02}$ & $1.4314 \times 10^{+04}$ \\
\hline${ }^{135} \mathrm{Ba}^{53+}$ & $3 / 2$ & 0.837943 & $3.5607 \times 10^{-02}$ & $-2.6223 \times 10^{-04}$ & $3.7640 \times 10^{-05}$ & $-2.5162 \times 10^{-04}$ & $3.5131 \times 10^{-02}$ & $3.5292 \times 10^{+04}$ \\
\hline${ }^{137} \mathrm{Ba}^{53+}$ & $3 / 2$ & 0.937365 & $3.9828 \times 10^{-02}$ & $-2.9435 \times 10^{-04}$ & $4.2102 \times 10^{-05}$ & $-2.8144 \times 10^{-04}$ & $3.9295 \times 10^{-02}$ & $3.1552 \times 10^{+04}$ \\
\hline${ }^{138} \mathrm{La}^{54+}$ & 5 & 3.713646 & $1.3941 \times 10^{-01}$ & $-1.0759 \times 10^{-03}$ & $1.5051 \times 10^{-04}$ & $-1.0065 \times 10^{-03}$ & $1.3748 \times 10^{-01}$ & $9.0186 \times 10^{+03}$ \\
\hline${ }^{139} \mathrm{La}^{54+}$ & $7 / 2$ & 2.783046 & $1.0854 \times 10^{-01}$ & $-8.3912 \times 10^{-04}$ & $1.1718 \times 10^{-04}$ & $-7.8366 \times 10^{-04}$ & $1.0703 \times 10^{-01}$ & $1.1584 \times 10^{+04}$ \\
\hline${ }^{141} \operatorname{Pr}^{56+}$ & $5 / 2$ & 4.2754 & $2.0045 \times 10^{-01}$ & $-1.6835 \times 10^{-03}$ & $2.2560 \times 10^{-04}$ & $-1.5095 \times 10^{-03}$ & $1.9748 \times 10^{-01}$ & $6.2782 \times 10^{+03}$ \\
\hline${ }^{143} \mathrm{Nd}^{57+}$ & $7 / 2$ & -1.065 & $5.0838 \times 10^{-02}$ & $-4.4606 \times 10^{-04}$ & $5.8398 \times 10^{-05}$ & $-3.9081 \times 10^{-04}$ & $5.0059 \times 10^{-02}$ & $2.4767 \times 10^{+04}$ \\
\hline${ }^{145} \mathrm{Nd}^{57+}$ & $7 / 2$ & -0.656 & $3.1311 \times 10^{-02}$ & $-2.7560 \times 10^{-04}$ & $3.5967 \times 10^{-05}$ & $-2.4070 \times 10^{-04}$ & $3.0830 \times 10^{-02}$ & $4.0215 \times 10^{+04}$ \\
\hline${ }^{147} \mathrm{Sm}^{59+}$ & $7 / 2$ & -0.8148 & $4.4373 \times 10^{-02}$ & $-4.2792 \times 10^{-04}$ & $5.3068 \times 10^{-05}$ & $-3.5520 \times 10^{-04}$ & $4.3643 \times 10^{-02}$ & $2.8409 \times 10^{+04}$ \\
\hline${ }^{149} \mathrm{Sm}^{59+}$ & $7 / 2$ & -0.6708 & $3.6527 \times 10^{-02}$ & $-3.5331 \times 10^{-04}$ & $4.3684 \times 10^{-05}$ & $-2.9239 \times 10^{-04}$ & $3.5925 \times 10^{-02}$ & $3.4512 \times 10^{+04}$ \\
\hline${ }^{151} \mathrm{Eu}^{60+}$ & $5 / 2$ & 3.4717 & $2.1188 \times 10^{-01}$ & $-2.1435 \times 10^{-03}$ & $2.5849 \times 10^{-04}$ & $-1.7302 \times 10^{-03}$ & $2.0827 \times 10^{-01}$ & $5.9531 \times 10^{+03}$ \\
\hline${ }^{153} \mathrm{Eu}^{60+}$ & $5 / 2$ & 1.533 & $9.3550 \times 10^{-02}$ & $-9.4915 \times 10^{-04}$ & $1.1413 \times 10^{-04}$ & $-7.6389 \times 10^{-04}$ & $9.1951 \times 10^{-02}$ & $1.3484 \times 10^{+04}$ \\
\hline${ }^{155} \mathrm{Gd}^{61+}$ & $3 / 2$ & -0.33726 & $2.4406 \times 10^{-02}$ & $-2.5758 \times 10^{-04}$ & $3.0366 \times 10^{-05}$ & $-2.0324 \times 10^{-04}$ & $2.3976 \times 10^{-02}$ & $5.1713 \times 10^{+04}$ \\
\hline${ }^{155} \mathrm{Gd}^{61+}$ & $3 / 2$ & -0.33726 & $2.4406 \times 10^{-02}$ & $-2.5758 \times 10^{-04}$ & $3.0366 \times 10^{-05}$ & $-2.0324 \times 10^{-04}$ & $2.3976 \times 10^{-02}$ & $5.1713 \times 10^{+04}$ \\
\hline${ }^{159} \mathrm{~Tb}^{62+}$ & $3 / 2$ & 2.014 & $1.5554 \times 10^{-01}$ & $-1.6961 \times 10^{-03}$ & $1.9733 \times 10^{-04}$ & $-1.3207 \times 10^{-03}$ & $1.5272 \times 10^{-01}$ & $8.1182 \times 10^{+03}$ \\
\hline${ }^{161} \mathrm{Dy}^{63+}$ & $5 / 2$ & -0.4803 & $3.5582 \times 10^{-02}$ & $-4.0916 \times 10^{-04}$ & $4.6022 \times 10^{-05}$ & $-3.0799 \times 10^{-04}$ & $3.4911 \times 10^{-02}$ & $3.5515 \times 10^{+04}$ \\
\hline${ }^{163} \mathrm{Dy}^{63+}$ & $5 / 2$ & 0.6726 & $4.9821 \times 10^{-02}$ & $-5.7442 \times 10^{-04}$ & $6.4440 \times 10^{-05}$ & $-4.3125 \times 10^{-04}$ & $4.8880 \times 10^{-02}$ & $2.5365 \times 10^{+04}$ \\
\hline${ }^{165} \mathrm{Ho}^{64+}$ & $7 / 2$ & 4.132 & $3.1082 \times 10^{-01}$ & $-3.7196 \times 10^{-03}$ & $4.0980 \times 10^{-04}$ & $-2.7422 \times 10^{-03}$ & $3.0477 \times 10^{-01}$ & $4.0682 \times 10^{+03}$ \\
\hline${ }^{165} \mathrm{Ho}^{64+}$ & $7 / 2$ & 4.173 & $3.1390 \times 10^{-01}$ & $-3.7565 \times 10^{-03}$ & $4.1386 \times 10^{-04}$ & $-2.7694 \times 10^{-03}$ & $3.0779 \times 10^{-01}$ & $4.0282 \times 10^{+03}$ \\
\hline${ }^{167} \operatorname{Er}^{65+}$ & $7 / 2$ & -0.56385 & $4.5195 \times 10^{-02}$ & $-5.6566 \times 10^{-04}$ & $6.0730 \times 10^{-05}$ & $-4.0633 \times 10^{-04}$ & $4.4284 \times 10^{-02}$ & $2.7998 \times 10^{+04}$ \\
\hline${ }^{169} \mathrm{Tm}^{66+}$ & $1 / 2$ & -0.2316 & $3.4629 \times 10^{-02}$ & $-4.4844 \times 10^{-04}$ & $4.7417 \times 10^{-05}$ & $-3.1721 \times 10^{-04}$ & $3.3911 \times 10^{-02}$ & $3.6562 \times 10^{+04}$ \\
\hline${ }^{171} \mathrm{Yb}^{67+}$ & $1 / 2$ & 0.49367 & $7.8611 \times 10^{-02}$ & $-1.0654 \times 10^{-03}$ & $1.0967 \times 10^{-04}$ & $-7.3357 \times 10^{-04}$ & $7.6921 \times 10^{-02}$ & $1.6118 \times 10^{+04}$ \\
\hline${ }^{173} \mathrm{Yb}^{67+}$ & $5 / 2$ & -0.67989 & $6.4948 \times 10^{-02}$ & $-8.8239 \times 10^{-04}$ & $9.0610 \times 10^{-05}$ & $-6.0608 \times 10^{-04}$ & $6.3550 \times 10^{-02}$ & $1.9510 \times 10^{+04}$ \\
\hline${ }^{175} \mathrm{Lu}^{68+}$ & $7 / 2$ & 2.2323 & $2.1619 \times 10^{-01}$ & $-3.0823 \times 10^{-03}$ & $3.0726 \times 10^{-04}$ & $-2.0549 \times 10^{-03}$ & $2.1136 \times 10^{-01}$ & $5.8659 \times 10^{+03}$ \\
\hline${ }^{176} \mathrm{Lu}^{68+}$ & 7 & 3.1692 & $2.8773 \times 10^{-01}$ & $-4.1069 \times 10^{-03}$ & $4.0892 \times 10^{-04}$ & $-2.7348 \times 10^{-03}$ & $2.8129 \times 10^{-01}$ & $4.4077 \times 10^{+03}$ \\
\hline${ }^{177} \mathrm{Hf}^{69+}$ & $7 / 2$ & 0.7935 & $8.1893 \times 10^{-02}$ & $-1.2032 \times 10^{-03}$ & $1.1855 \times 10^{-04}$ & $-7.9274 \times 10^{-04}$ & $8.0016 \times 10^{-02}$ & $1.5495 \times 10^{+04}$ \\
\hline${ }^{179} \mathrm{Hf}^{69+}$ & $9 / 2$ & -0.6409 & $6.4296 \times 10^{-02}$ & $-9.4686 \times 10^{-04}$ & $9.3074 \times 10^{-05}$ & $-6.2240 \times 10^{-04}$ & $6.2820 \times 10^{-02}$ & $1.9736 \times 10^{+04}$ \\
\hline${ }^{180} \mathrm{Ta}^{70+}$ & 9 & 4.77 & $4.8435 \times 10^{-01}$ & $-7.3630 \times 10^{-03}$ & $7.1404 \times 10^{-04}$ & $-4.7742 \times 10^{-03}$ & $4.7292 \times 10^{-01}$ & $2.6217 \times 10^{+03}$ \\
\hline${ }^{181} \mathrm{Ta}^{70+}$ & $7 / 2$ & 2.3705 & $2.6059 \times 10^{-01}$ & $-3.9659 \times 10^{-03}$ & $3.8416 \times 10^{-04}$ & $-2.5686 \times 10^{-03}$ & $2.5444 \times 10^{-01}$ & $4.8729 \times 10^{+03}$ \\
\hline${ }^{183} \mathrm{~W}^{71+}$ & $1 / 2$ & 0.117785 & $2.4112 \times 10^{-02}$ & $-3.8423 \times 10^{-04}$ & $3.6197 \times 10^{-05}$ & $-2.4200 \times 10^{-04}$ & $2.3522 \times 10^{-02}$ & $5.2710 \times 10^{+04}$ \\
\hline${ }^{185} \mathrm{Re}^{72+}$ & $5 / 2$ & 3.1871 & $4.1693 \times 10^{-01}$ & $-6.8741 \times 10^{-03}$ & $6.3723 \times 10^{-04}$ & $-4.2599 \times 10^{-03}$ & $4.0643 \times 10^{-01}$ & $3.0506 \times 10^{+03}$ \\
\hline${ }^{187} \mathrm{Re}^{72+}$ & $5 / 2$ & 3.2197 & $4.2111 \times 10^{-01}$ & $-6.9581 \times 10^{-03}$ & $6.4363 \times 10^{-04}$ & $-4.3027 \times 10^{-03}$ & $4.1050 \times 10^{-01}$ & $3.0203 \times 10^{+03}$ \\
\hline${ }^{187} \mathrm{Os}^{73+}$ & $1 / 2$ & 0.064652 & $1.5001 \times 10^{-02}$ & $-2.5809 \times 10^{-04}$ & $2.3340 \times 10^{-05}$ & $-1.5602 \times 10^{-04}$ & $1.4610 \times 10^{-02}$ & $8.4863 \times 10^{+04}$ \\
\hline${ }^{189} \mathrm{Os}^{73+}$ & $3 / 2$ & 0.659933 & $1.0206 \times 10^{-01}$ & $-1.7597 \times 10^{-03}$ & $1.5880 \times 10^{-04}$ & $-1.0615 \times 10^{-03}$ & $9.9399 \times 10^{-02}$ & $1.2473 \times 10^{+04}$ \\
\hline${ }^{191} \operatorname{Ir}{ }^{74+}$ & $3 / 2$ & 0.1507 & $2.4813 \times 10^{-02}$ & $-4.4391 \times 10^{-04}$ & $3.9297 \times 10^{-05}$ & $-2.6268 \times 10^{-04}$ & $2.4146 \times 10^{-02}$ & $5.1348 \times 10^{+04}$ \\
\hline${ }^{193} \operatorname{Ir}^{74+}$ & $3 / 2$ & 0.1637 & $2.6949 \times 10^{-02}$ & $-4.8310 \times 10^{-04}$ & $4.2679 \times 10^{-05}$ & $-2.8528 \times 10^{-04}$ & $2.6223 \times 10^{-02}$ & $4.7281 \times 10^{+04}$ \\
\hline${ }^{195} \mathrm{Pt}^{75+}$ & $1 / 2$ & 0.60952 & $1.6028 \times 10^{-01}$ & $-2.9739 \times 10^{-03}$ & $2.5834 \times 10^{-04}$ & $-1.7268 \times 10^{-03}$ & $1.5584 \times 10^{-01}$ & $7.9560 \times 10^{+03}$ \\
\hline${ }^{197} \mathrm{Au}^{76+}$ & $3 / 2$ & 0.148158 & $2.7629 \times 10^{-02}$ & $-5.3617 \times 10^{-04}$ & $4.5318 \times 10^{-05}$ & $-3.0293 \times 10^{-04}$ & $2.6835 \times 10^{-02}$ & $4.6202 \times 10^{+04}$ \\
\hline${ }^{199} \mathrm{Hg}^{77+}$ & $1 / 2$ & 0.505885 & $1.5065 \times 10^{-01}$ & $-3.0356 \times 10^{-03}$ & $2.5141 \times 10^{-04}$ & $-1.6807 \times 10^{-03}$ & $1.4618 \times 10^{-01}$ & $8.4815 \times 10^{+03}$ \\
\hline${ }^{201} \mathrm{Hg}^{77+}$ & $3 / 2$ & -0.560226 & $1.1120 \times 10^{-01}$ & $-2.2449 \times 10^{-03}$ & $1.8557 \times 10^{-04}$ & $-1.2406 \times 10^{-03}$ & $1.0790 \times 10^{-01}$ & $1.1491 \times 10^{+04}$ \\
\hline${ }^{203} \mathrm{Tl}^{78+}$ & $1 / 2$ & 1.622258 & $5.1412 \times 10^{-01}$ & $-1.0789 \times 10^{-02}$ & $8.7292 \times 10^{-04}$ & $-5.8362 \times 10^{-03}$ & $4.9837 \times 10^{-01}$ & $2.4878 \times 10^{+03}$ \\
\hline${ }^{205} \mathrm{Tl}^{78+}$ & $1 / 2$ & 1.638215 & $5.1906 \times 10^{-01}$ & $-1.0913 \times 10^{-02}$ & $8.8131 \times 10^{-04}$ & $-5.8923 \times 10^{-03}$ & $5.0314 \times 10^{-01}$ & $2.4642 \times 10^{+03}$ \\
\hline${ }^{207} \mathrm{~Pb}^{79+}$ & $1 / 2$ & 0.58219 & $1.9635 \times 10^{-01}$ & $-4.2943 \times 10^{-03}$ & $3.3912 \times 10^{-04}$ & $-2.2677 \times 10^{-03}$ & $1.9012 \times 10^{-01}$ & $6.5213 \times 10^{+03}$ \\
\hline${ }^{207} \mathrm{~Pb}^{79+}$ & $1 / 2$ & 0.592583 & $1.9985 \times 10^{-01}$ & $-4.3710 \times 10^{-03}$ & $3.4518 \times 10^{-04}$ & $-2.3082 \times 10^{-03}$ & $1.9352 \times 10^{-01}$ & $6.4069 \times 10^{+03}$ \\
\hline${ }^{209} \mathrm{Bi}^{80+}$ & $9 / 2$ & 4.1106 & $8.1967 \times 10^{-01}$ & $-1.8675 \times 10^{-02}$ & $1.4400 \times 10^{-03}$ & $-9.6310 \times 10^{-03}$ & $7.9280 \times 10^{-01}$ & $1.5639 \times 10^{+03}$ \\
\hline${ }^{235} \mathrm{U}^{89+}$ & $7 / 2$ & -0.38 & $1.3721 \times 10^{-01}$ & $-4.4915 \times 10^{-03}$ & $2.7948 \times 10^{-04}$ & $-1.8780 \times 10^{-03}$ & $1.3112 \times 10^{-01}$ & $9.4560 \times 10^{+03}$ \\
\hline
\end{tabular}

Table 2. (Continued) 


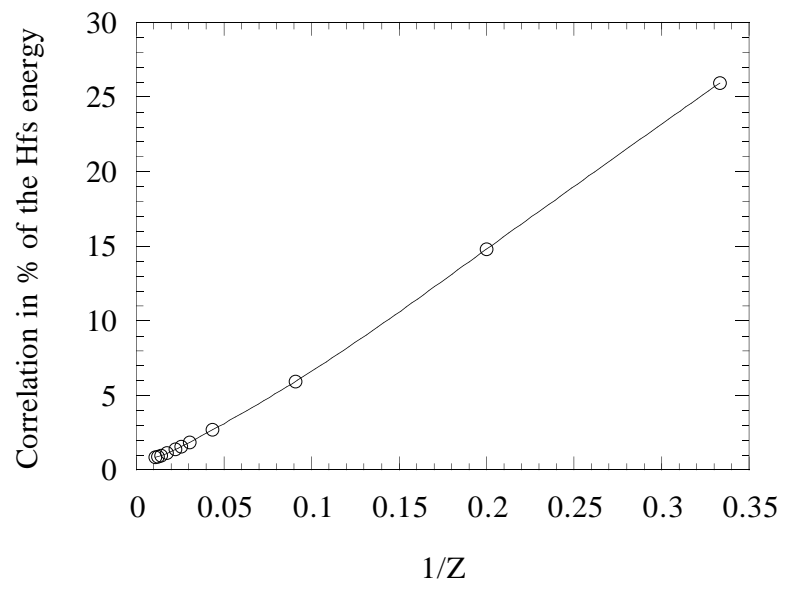

Fig. 6. Increase with $\mathrm{Z}$ of the $1 \mathrm{~s} 2 \mathrm{~s} 3 \mathrm{~s}$ configuration relative contribution to the correlation hyperfine energy.

reduces it by about $4 \%$ at $Z=92$. The electron-electron Coulomb interaction dominates by two orders of magnitude all other corrections at low $Z$. Coulomb correlation stays an essential contribution at high- $Z$, but it is overcome around $Z=55$ by both Bohr-Weisskopf and radiative corrections which increase strongly with the atomic number.

The change in hyperfine energy due to the Coulomb correlation is very large for small values of atomic number $Z$ and decreases roughly as $1 / Z$ when $Z$ increases. For illustrate this point, we have plotted (Fig. 6) the $1 \mathrm{~s} 2 \mathrm{~s} 3 \mathrm{~s}$ configuration relative contribution to the correlation hyperfine energy. This represents more than $25 \%$ of the total hyperfine transition energy at $Z=3$, about $15 \%$ at $Z=5$ and just $6 \%$ at $Z=11$. As we have seen in Sec. 3, the $1 s 2 s 3 s J=1 / 2$ configuration is the most important contribution to the correlation. Its relative contribution to the total correlation energy varies very slowly with $Z$ except for very low $Z$. For $Z \geq 45$ no other configuration contributes by more than $1 \%$. This is a very interesting feature of heavy lithium-like ions, enabling to reach high accuracy with a limited set of configurations, particularly for the more advanced calculations with negative continuum, for which it is very hard to reach convergence. The situation is very different for neutral $\mathrm{Li}$ or light ions, for which a very large number of configurations is needed, and convergence as a function of the number of configurations is both slow and highly oscillatory [43].

Magnetic correlations (Fig. 4) and negative continuum contributions (Fig. 7) are plotted in Fig. 8. The ratio of these corrections to the hyperfine energy is small compared to the other corrections (Coulomb correlation, Bohr-Weisskopf or QED) even if they become more important at high $Z(\approx 0.15 \%)$. We have regrouped these terms in Fig. 5 but we can follow their relative importance in Fig. 8. To get their values we do 4 different calculations. In the first two ones the magnetic interaction is neglected. One calculation is performed without projection operators, and one is done with them. Both calculations are repeated with a self-consistent magnetic interaction. We can

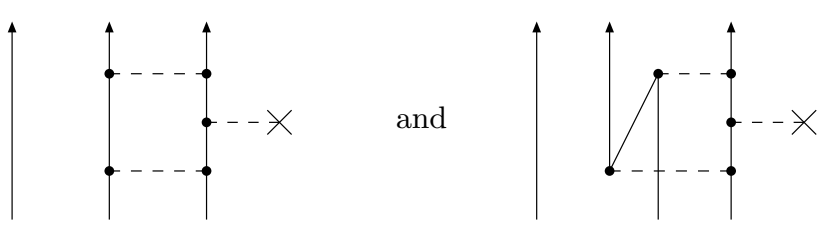

Fig. 7. Feynman diagrams with negative continuum on the magnetic part of the Gaunt interaction contribution to the hyperfine structure of a three electron ion. When including projection operators, the right diagram is suppressed. We include the same diagrams for the Coulomb interaction.

thus obtain by differences the mixed Coulomb-negative continuum term and the Gaunt-negative continuum term. At low $Z$ the Gaunt-negative continuum mixed contribution is much larger than the Coulomb-negative continuum and both contributions approximatively cancel at high $Z$. These two terms and Gaunt correlation are small but not negligible if one needs a high accuracy calculation.

Finally we examine the precision of our calculation for low- $Z$. Because of the way we treat magnetic corrections and negative continuum, we are limited in the number of configurations we can include in the calculation. Calculations with thousands of $L S$ configurations have been performed for $\mathrm{Li}$ and light ions and it is instructive to compare our results with both experiment and theoretical calculations. For instance, our hyperfine structure constant $A_{1 / 2}$ for ${ }^{7} \mathrm{Li}$ and ${ }^{9} \mathrm{Be}$ are respectively $390.804 \mathrm{MHz}$ and $-624.113 \mathrm{MHz}$ while the most precise measurements give $401.7520433(5) \mathrm{MHz}$ and $-625.008837048(10) \mathrm{MHz}$ respectively (Ref. [44] and references therein). The difference between theory and experiment is thus $2.73 \%$ for ${ }^{7} \mathrm{Li}$ and $0.14 \%$ for ${ }^{9} \mathrm{Be}$, which correspond to a difference in correlation of $14.7 \%$ and $7.3 \%$ respectively. If we add to our calculations all the possible configurations containing

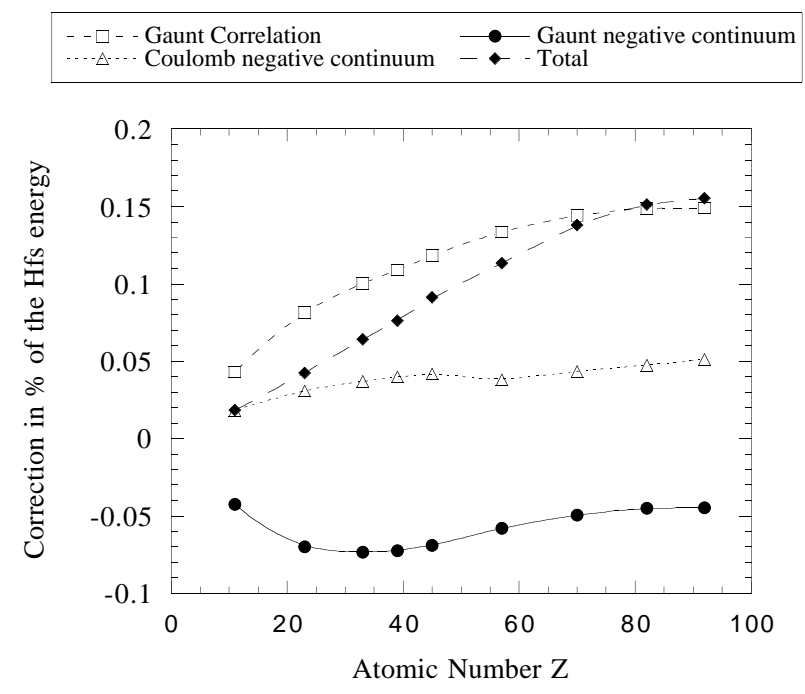

Fig. 8. Relative contribution of each term: magnetic (Gaunt) correlation, Coulomb-negative continuum, Gauntnegative continuum and total correction of this three corrections. 
a $4 s$ orbital, we find $A_{1 / 2}=376.309 \mathrm{MHz}(Z=3)$ and $A_{1 / 2}=-625.008 \mathrm{MHz}(Z=4)$. The difference with the experimental results increases. The reason is the slow, oscillatory convergence as a function of the number of configuration at low $Z$ [43]. We have estimated the uncertainty on our correlation contribution at high $Z$ with a fit of this correction adjusted on the ${ }^{7} \mathrm{Li}$ and ${ }^{9} \mathrm{Be}$ experimental values. We find $\approx 5 \%$ at $Z=5,1 \%$ at $Z=46$ and $0.1 \%$ at $Z=92$.

\subsection{Bohr-Weisskopf effects}

Because of its large size and large uncertainty, and because it does not really depend on the atomic part of the calculation we discuss the Bohr-Weisskopf effect separately. In order to assess the reliability of our very simple Bohr-Weisskopf model we compare calculations we performed for the ground state of hydrogen-like ion with experiment and other calculations in Table 3. The comparison with other calculations can also provide a check of our self-consistent calculation for vacuum polarization correction. Our vacuum polarization (Uëhling SC) is in good agreement with the equivalent terms in other calculations, which shows that contributions with two and more loops, which we include, but which are not included in direct $\mathrm{QED}$ calculations, have a very small contribution as expected (they are of order $\alpha$ compared to the one loop correction). The difference between our vacuum polarization (Uëhling SC) and the other calculations [2, $3,28,45]$ (normalized to our results) is smaller than $2 \%$ for ${ }^{165} \mathrm{Ho}^{66+}$ and ${ }^{187} \mathrm{Re}^{74+}, 5 \%$ for ${ }^{207} \mathrm{~Pb}^{81+}$ and $4 \%$ for ${ }^{209} \mathrm{Bi}^{82+}$. All these vacuum polarization calculations $[2$, $3,28,45]$ include the Wichmann-Kroll contribution while ours include only Uehling approximation. The corresponding variations on the total theoretical result are respectively $0.005 \%, 0.007 \%, 0.024 \%$ and $0.019 \%$. In conclusion our QED term (vacuum polarization and self-energy corrections) are in agreement with all other calculations including recent results Ref. [46]. Yet the different calculations of the Bohr-Weisskopf effect are not in agreement (variation of about 10\% to 50\%). There is no clear trend in the comparison between experiment and theory enabling to select a theoretical model for the Bohr-Weisskopf effect, and our very simple model is completely equivalent to more sophisticated ones.

For lithium-like ions the lack of experimental data at high- $Z$ does not allow to do a detailed comparison. We can only compare our calculation with the measurement of the hyperfine transition in lithium-like Bi [47]. Our theoretical value is slightly outside experimental uncertainty. We find a transition energy of $792.804 \mathrm{meV}$ to be compared to an experimental value of $820(26) \mathrm{meV}$ (Table 4). In a first paper V.M. Shabaev et al. [48] found a similar result with $800(7) \mathrm{meV}$. In a second paper [8,9], after a reevaluation of some terms, they give 796.9(2) meV (inside the error bar) which differs from our calculation by $0.5 \%$. One can compare more precisely the different terms between Ref. [8,9] and our result. Of course we are in agreement for the point nucleus value of $958.49 \mathrm{meV}$.

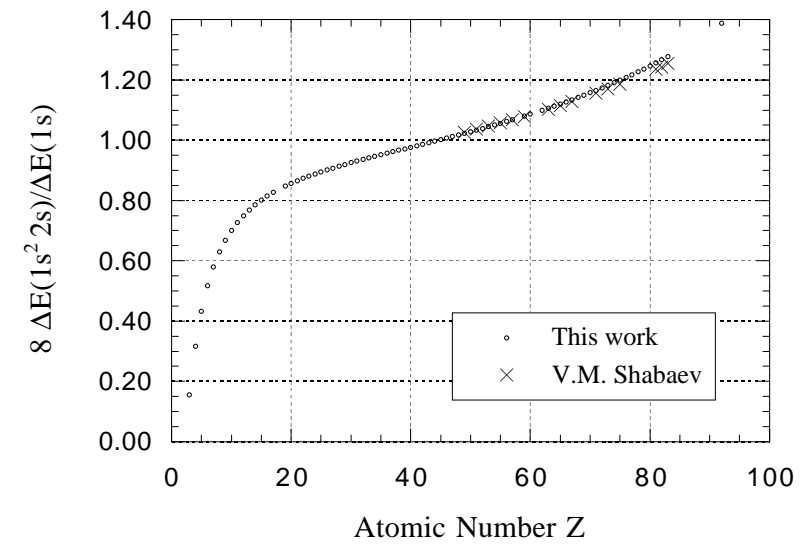

Fig. 9. Ratio between hyperfine transition energy in Hydrogen-like and Lithium-like ions. V.M. Shabaev is Ref. [8].

Our nuclear size correction is $-108.42 \mathrm{meV}$ for a Ref. [8, 9] value of $-113.8 \mathrm{meV}$. This last correction $-113.8 \mathrm{meV}$ does not include the nuclear-size correction of $4.43 \mathrm{meV}$ to the inter-electronic interaction effect which is in the total inter-electronic interaction contribution of $-29.45 \mathrm{meV}$ in Ref. $[8,9]$. The total resulting difference for the nuclear size correction appears to be $.95 \mathrm{meV}$. The Dirac-Fock contribution $(-43.02 \mathrm{meV})$ is the difference between a calculation with MCDF orbitals and hydrogen-like orbitals. If we add the Coulomb correlation to this term, we obtain $-35.8 \mathrm{meV}$. This corresponds to the Coulomb part $(-36 \mathrm{meV})$ of the inter-electronic interaction in $[8,9]$ (see also $[47,48])$. For the total value, we must compare our Dirack-Fock term, correlation terms and negative continuum terms $(-34.53 \mathrm{meV})$ to Ref. $[8,9]$ value $(-29.45 \mathrm{meV})$. We find a difference of $5.08 \mathrm{meV}$ which corresponds mostly to the nuclear size correction on the magnetic correlation (4.43 meV see above) and QED part for $0.38 \mathrm{meV}$. Thus our total QED term is $-4.06 \mathrm{meV}$ for $-4.44 \mathrm{meV}$ in Shabaev (One-electron QED -4.6 meV and Inter-electronic interaction QED $0.16 \mathrm{meV}$ ). This is because we have not separate the vacuum polarization due to the introduction of the Gaunt potential in the self-consistent process. In fine, if we use the same Bohr-Weisskopf correction our total results differ only by $0.68 \mathrm{meV}(0.95 \mathrm{meV}$ on the nuclear size correction and $-0.27 \mathrm{meV}$ on correlations terms) which correspond to $0.09 \%$ !

To obtain a better evaluation of the $2 s$ Bohr-Weisskopf correction, it is possible to use the method proposed in Ref. [8], using experimental data for hydrogen-like ions. For this reason, we give in appendix the value of the ground-state hyperfine splitting for hydrogen-like ions (Table 5), with the same details as for lithium-like ions (Table 2). This method make use of the normalized ratio $\eta=8 \Delta E_{L i} / \Delta E_{H}$ of the lithium-like to hydrogen-like energy. The ratio that we obtain is compared to the one of Ref. [8] on Fig. 9. Agreement between both calculation is very good except for the highest $Z$. Finally we plotted the total hyperfine splitting in $\mu m$ as a function of the atomic number $Z$ for hydrogen-like and lithium-like ions on Fig. 10. They are two area for which spectroscopy can 
be performed : the first above $10^{3} \mu \mathrm{m}$ by hyper-frequence spectroscopy $(3 \leq Z \leq 19)$ and the second below $10 \mu m$ by laser spectroscopy $(48 \leq Z \leq 92)$. Among this two values, experimental measurement are more complicated.

\section{Conclusion}

In this work we have studied relativistic effects and some radiative corrections on the ground-state hyperfine structure of three-electron ions with the MCDF method. We have also proposed a simple model for the Bohr-Weisskopf effect.

We have investigated the role of the magnetic interaction and negative energy continuum in the correlation. We have shown that contrary to what has been observed in the case of the relativistic $M 1$ in helium the negative energy continuum plays a small role and is sizeable only at high- $Z$. The magnetic correlation correction is small compared to the Coulomb correlation, even at high- $Z$.

The effect of Feynman diagrams with vacuum polarization loops modifying the wave function has been computed to all orders by including the vacuum polarization potential into the MCDF procedure. The result of this calculation is in good agreement with perturbative calculation.

Finally we showed that our very simple model for the Bohr-Weisskopf effect can be used as reliably (or unreliably) as model based on nuclear model calculations. Very little advance can be expected in our understanding of the hyperfine structure in heavy ions until a much better procedure to handle the the Bohr-Weisskopf effect is proposed.

\section{References}

1. I. Klaft, S. Borneis, T. Engel, B. Fricke, R. Grieser, G. Huber, T. Khl, D. Marx, R. Neumann, S. Schrder, P. Seelig, L. Vlker, Phys. Rev. Lett. 73, 2425 (1994).

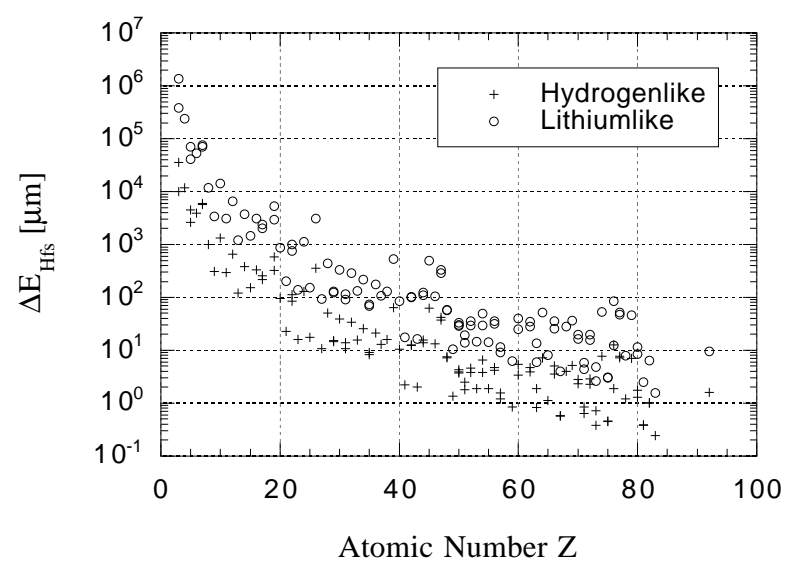

Fig. 10. The hyperfine splitting energies in $\mu m$ for hydrogenlike and lithium-like ions in function of the atomic number $Z$.
2. P. Seelig, S. Borneis, A. Dax, T. Engel, S. Faber, M. Gerlach, C. Holbrow, G. Huber, T. Khl, D. Marx, K. Meier, P. Merz, W. Quint, F. Schmitt, L. Vlker, H. Winter, M. Wrtz, K. Beckert, B. Franzke, F. Nolden, H. Reich, M. Steck, T. Winkler, Phys. Rev. Lett. 81, 4824 (1998).

3. J. R. C. López-Urrutia, P. Beiersdorfer, D. W. Savin, K. Widmann, Phys. Rev. Lett. 77, 826 (1996).

4. J. R. C. López-Urrutia, P. Beiersdorfer, K. Widmann, B. B. Birket, A. M. Martenson-Pendrill, M. G. H. Gustavson, Phys. Rev. A 57, 879 (1998).

5. A. Bohr V. F. Weisskopf, Phys. Rev. 77, 94 (1950).

6. M. Tomaselli, S. M. Schneider, E. Kankeleit, T. Khl, Phys. Rev. C 51, 2989 (1995).

7. L. N. Labzowsky, W. R. Johnson, G. Soff, S. M. Schneider, Phys. Rev. A 51, 4597 (1995).

8. V. M. Shabaev, M. B. Shabaeva, I. I. Tupitsyn, V. A. Yerokhin, A. N. Artemyev, T. Khl, M. Tomaselli, Phys. Rev. A 57, 149 (1998).

9. V. M. Shabaev, M. B. Shabaeva, I. I. Tupitsyn, V. A. Yerokhin, A. N. Artemyev, T. Khl, M. Tomaselli, Phys. Rev. A 58, 1610 (1998).

10. C. S. Wood, S. C. Bennet, D. Cho, B. P. Masterson, J. L. Roberts, C. E. Tanner, C. E. Wieman, Science 275, 1759 (1997).

11. P. Indelicato, Phys. Rev. A 51, 1132 (1995).

12. P. Indelicato, Phys. Rev. Lett. 77, 3323 (1996).

13. A. Derevianko, I. M. Savukov, W. R. Johnson, Phys. Rev. A 58, 4453 (1998).

14. J. P. Desclaux, Computer Physics Communication 9, 31 (1975).

15. I. P. Grant, Adv. Phys. 19, 747 (1970).

16. G. E. Brown D. E. Ravenhall, Proc. R. Soc. London, Ser A 208, 552 (1951).

17. J. Sucher, Phys. Rev. A 22, 348 (1980).

18. M. H. Mittleman, Phys. Rev. A 24, 1167 (1981).

19. J. P. Desclaux, in Methods and Techniques in Computational Chemistry, edited by E. Clementi (STEF, Cagliary, 1993), Vol. A.

20. A. R. Edmonds, Angular Momentum in Quantum Mechanics (Princeton University Press, Princeton, New Jersey, 1974).

21. I. Lindgren A. Rosn, Case Stud. At. Phys. 4, 93 (1974).

22. A. I. Akhiezer V. B. Berestetskii, Quantum Electrodynamics (Interscience, New York, 1965).

23. K. T. Cheng W. J. Childs, Phys. Rev. A 31, 2775 (1985).

24. W. R. Johnson G. Soff, At. Data Nucl. Data Tables 33, 405 (1985).

25. P. Indelicato E. Lindroth, Phys. Rev. A 46, 2426 (1992).

26. A. J. Freeman, M. Weinert, J. P. Desclaux, J. V. Mallow, Journal of Magnetism and Magnetic Materials 22, L1 (1980).

27. S. M. Schneider, W. Greiner, G. Soff, Phys. Rev. A 50, 118 (1994).

28. H. Persson, S. M. Schneider, W. Greiner, G. Soff, I. Lindgren, Phys. Rev. Lett. 76, 1433 (1996).

29. S. A. Blundell, K. T. Cheng, J. Sapirstein, Phys. Rev. A 55, 1857 (1997).

30. S. Klarsfeld, Phys. Lett. 66B, 86 (1977).

31. R. C. Barrett, S. J. Brodsky, G. W. Erickson, M. H. Goldhaber, Phys. Rev. 166, 1589 (1968).

32. L. W. Fullerton G. A. Rinker, Phys. Rev. A 13, 1283 (1976). 
33. W. H. Press, B. P. Flannery, S. A. Teukolsky, W. T. Vetterling, Numerical Recipes (Cambridge University Press, Cambridge, 1986), p. 818.

34. M. Abramowitz I. A. Stegun, in Handbook of mathematical functions (Dover, New York, 1965), p. 1046.

35. P. J. Mohr, G. Plunien, G. Soff, Phy. Rep. 293, 227 (1998).

36. K. Pachucki, Phys. Rev. A 54, 1994 (1996).

37. S. G. Karshenboim, Z. Phys. D 36, 11 (1996).

38. P. Indelicato J. P. Desclaux, Phys. Rev. A 42, 5139 (1990).

39. J. Sapirstein, K. T. Cheng, M. H. Chen, Phys. Rev. A 59, 259 (1999).

40. P. Raghavan, At. Data Nucl. Data Tables 42, 189 (1989).

41. G. Nachtsheim, Ph.D. thesis, Universitat Bonn, 1980.

42. L. K. Peker, Nuc. Data Sheets 50, 137 (1987).

43. J. Bieron, P. Jönsson, C. F. Fischer, Phys. Rev. A 53, 1 (1996).

44. X.-X. Guan Z.-W. Wang, Eur. Phys. J. D 2, 21 (1998).

45. V. M. Shabaev, M. Tomaselli, T. Khl, A. N. Artemyev, V. A. Yerokhin, Phys. Rev. A 56, 252 (1997).

46. P. Sunnergren, H. Persson, S. Salomonson, S. M. Schneider, I. Lindgren, G. Soff, Phys. Rev. A 58, 1055 (1998).

47. P. Beiersdorfer, A. Osterheld, J. Scofield, J. C. LpezUrrutia, K. Widmann, Phys. Rev. Lett. 80, 3022 (1998).

48. M. B. Shabaeva V. M. Shabaev, Phys. Rev. A 52, 2811 (1995). 


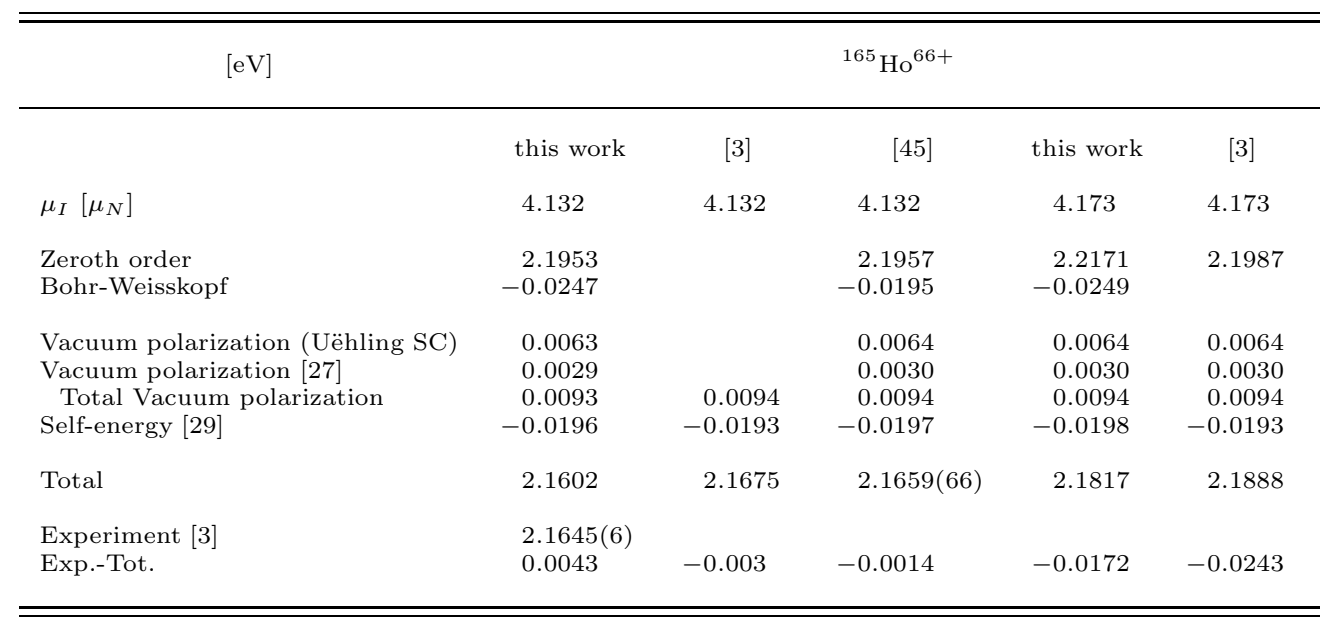

\begin{tabular}{lrrr}
\hline \multicolumn{1}{c}{$[\mathrm{eV}]$} & \multicolumn{2}{c}{${ }^{185} \mathrm{Re}^{74+}$} & ${ }^{187} \mathrm{Re}^{74+}$ \\
& & & \\
& this work & {$[45]$} & this work \\
& & & \\
& 3.1871 & 3.1871 & 3.2197 \\
$\mu_{I}\left[\mu_{N}\right]$ & & & \\
& 2.7981 & 2.7977 & 2.8262 \\
Zeroth order & -0.0429 & -0.0341 & -0.0434 \\
Bohr-Weisskopf & & & \\
& 0.0103 & 0.0105 & 0.0104 \\
Vacuum polarization (Uëhling SC) & 0.0043 & 0.0044 & 0.0044 \\
Vacuum polarization [27] & 0.0146 & 0.0149 & 0.0148 \\
$\quad$ Total Vacuum polarization & -0.0290 & -0.0290 & -0.0293 \\
Self-energy [29] & & & \\
Total & 2.7408 & $2.749(10)$ & 2.7683 \\
Experiment [4] & $2.719(2)$ & & $2.745(2)$ \\
Exp.-Tot. & -0.0218 & -0.030 & -0.0233 \\
& & & \\
\hline \hline
\end{tabular}

Table 3. Comparison between different theoretical calculations and experimental values in the hydrogen-like ions. 


\begin{tabular}{|c|c|c|c|c|c|}
\hline \multirow[t]{2}{*}[\mathrm{eV}]{} & \multicolumn{5}{|c|}{${ }^{207} \mathrm{~Pb}^{81+}$} \\
\hline & this work & {$[2]$} & this work & {$[28]$} & {$[45]$} \\
\hline$\mu_{I}\left[\mu_{N}\right]$ & 0.58219 & 0.58219 & 0.592583 & 0.592583 & 0.592583 \\
\hline Zeroth order & 1.2529 & 1.2566 & 1.2754 & $1.2752(2)$ & 1.2751 \\
\hline Bohr-Weisskopf & -0.0252 & -0.0400 & -0.0257 & -0.0340 & -0.0534 \\
\hline Vacuum polarization (Uëhling SC) & 0.0057 & & 0.0058 & 0.0061 & 0.0059 \\
\hline Vacuum polarization $[27]$ & 0.0022 & & 0.0022 & 0.0022 & 0.0023 \\
\hline Total Vacuum polarization & 0.0079 & 0.0082 & 0.0080 & 0.0083 & 0.0082 \\
\hline Self-energy $[29]$ & -0.0147 & -0.0142 & -0.0150 & -0.0148 & -0.0150 \\
\hline Total & 1.2208 & $1.2105(24)$ & 1.2427 & 1.2347 & $1.215(5)$ \\
\hline Experiment [2] & $1.2159(2)$ & & & & \\
\hline Exp.-Tot. & -0.0049 & 0.0054 & -0.0268 & -0.0188 & -0.0009 \\
\hline
\end{tabular}

\begin{tabular}{lccc}
\hline \multicolumn{1}{c}{$[\mathrm{eV}]$} & & & \\
& & & \\
& & & \\
& & & \\
& & & \\
& this work & {$[45]$} & {$[28]$} \\
& & & 4.1106 \\
$\mu_{I}\left[\mu_{N}\right]$ & 4.1106 & 4.1106 & $5.1917(10)$ \\
& & & $-0.107(7)$ \\
Zeroth order & 5.1908 & 5.1906 & 0.0253 \\
Bohr-Weisskopf & -0.1087 & -0.0612 & 0.0093 \\
& & & 0.0346 \\
Vacuum polarization (Uëhling SC) & 0.0243 & 0.0250 & -0.0614 \\
Vacuum polarization [27] & 0.0093 & 0.0093 & $5.058(8)$ \\
$\quad$ Total Vacuum polarization & 0.0335 & 0.0343 & \\
Self-energy [29] & -0.0619 & -0.0621 & -0.026 \\
Total & 5.0537 & $5.101(27)$ & \\
Experiment [1] & $5.0840(8)$ & & \\
Exp.-Tot. & 0.0303 & -0.017 & \\
& & & \\
\hline \hline
\end{tabular}

Table 3. (Continued) 


\begin{tabular}{lr}
\hline \hline \multicolumn{2}{c}{${ }^{209} \mathrm{Bi}^{80+}$} \\
\\
\hline Point nucleus (Hyd. orb.) & 958.49 \\
Dirac-Fock correction & -43.02 \\
Nuclear size correction & -108.42 \\
& \\
Bohr-Weisskopf & -18.68 \\
Correlations (Coulomb) & 7.22 \\
Correlations (magnetic) & 1.21 \\
Negative continuum (Coulomb) & 0.42 \\
Negative continuum (magnetic) & -0.36 \\
Vacuum polarization (Uëhling SC) & 4.13 \\
Vacuum polarization [27] & 1.44 \\
Self-energy [29] & -9.63 \\
Total & 792.80 \\
Experiment [47] & $820(26)$ \\
\hline \hline
\end{tabular}

Table 4. Value of the ground state hyperfine splitting of ${ }^{209} \mathrm{Bi}^{80+}$ (in $\mathrm{meV}$ ). 


\begin{tabular}{|c|c|c|c|c|c|c|c|c|}
\hline Label & $I$ & $\mu_{I}$ & $\Delta E_{H}^{H f s}$ & $\Delta E_{H}^{B W}$ & $V P_{H}^{h f s}$ & $S E_{H}$ & $\Delta E_{H}$ & $\lambda_{H}$ \\
\hline${ }^{6} \mathrm{Li}^{2+}$ & 1 & 0.8220473 & $3.5044 \times 10^{-05}$ & $-3.3367 \times 10^{-09}$ & $1.9953 \times 10^{-09}$ & $2.5040 \times 10^{-08}$ & $3.5068 \times 10^{-05}$ & $3.5356 \times 10^{+07}$ \\
\hline${ }^{7} \mathrm{Li}^{2+}$ & $3 / 2$ & 3.2564268 & $1.2340 \times 10^{-04}$ & $-1.2156 \times 10^{-08}$ & $7.0259 \times 10^{-09}$ & $8.8172 \times 10^{-08}$ & $1.2348 \times 10^{-04}$ & $1.0041 \times 10^{+07}$ \\
\hline${ }^{9} \mathrm{Be}^{3+}$ & $3 / 2$ & -1.177492 & $1.0582 \times 10^{-04}$ & $-1.4665 \times 10^{-08}$ & $8.0059 \times 10^{-09}$ & $5.9268 \times 10^{-08}$ & $1.0587 \times 10^{-04}$ & $1.1711 \times 10^{+07}$ \\
\hline${ }^{10} \mathrm{~B}^{4+}$ & 3 & 1.800645 & $2.7674 \times 10^{-04}$ & $-4.4669 \times 10^{-08}$ & $2.6086 \times 10^{-08}$ & $1.1206 \times 10^{-07}$ & $2.7683 \times 10^{-04}$ & $4.4786 \times 10^{+06}$ \\
\hline${ }^{11} \mathrm{~B}^{4+}$ & $3 / 2$ & 2.688649 & $4.7225 \times 10^{-04}$ & $-7.8116 \times 10^{-08}$ & $4.4515 \times 10^{-08}$ & $1.9122 \times 10^{-07}$ & $4.7241 \times 10^{-04}$ & $2.6245 \times 10^{+06}$ \\
\hline${ }^{13} \mathrm{C}^{5+}$ & $1 / 2$ & 0.702412 & $3.2005 \times 10^{-04}$ & $-6.6670 \times 10^{-08}$ & $3.6089 \times 10^{-08}$ & $7.9838 \times 10^{-08}$ & $3.2010 \times 10^{-04}$ & $3.8734 \times 10^{+06}$ \\
\hline${ }^{14} \mathrm{~N}^{6+}$ & 1 & 0.403761 & $2.1931 \times 10^{-04}$ & $-5.4423 \times 10^{-08}$ & $2.8766 \times 10^{-08}$ & $2.0622 \times 10^{-08}$ & $2.1931 \times 10^{-04}$ & $5.6534 \times 10^{+06}$ \\
\hline${ }^{15} \mathrm{~N}^{6+}$ & $1 / 2$ & -0.283189 & $2.0509 \times 10^{-04}$ & $-5.1827 \times 10^{-08}$ & $2.6901 \times 10^{-08}$ & $1.9285 \times 10^{-08}$ & $2.0509 \times 10^{-04}$ & $6.0454 \times 10^{+06}$ \\
\hline${ }^{17} \mathrm{O}^{7+}$ & $5 / 2$ & -1.89379 & $1.2297 \times 10^{-03}$ & $-3.7844 \times 10^{-07}$ & $1.8382 \times 10^{-07}$ & $-7.5211 \times 10^{-08}$ & $1.2294 \times 10^{-03}$ & $1.0085 \times 10^{+06}$ \\
\hline${ }^{19} \mathrm{~F}^{8+}$ & $1 / 2$ & 2.628868 & $4.0559 \times 10^{-03}$ & $-1.4858 \times 10^{-06}$ & $6.8022 \times 10^{-07}$ & $-8.7598 \times 10^{-07}$ & $4.0542 \times 10^{-03}$ & $3.0582 \times 10^{+05}$ \\
\hline${ }^{21} \mathrm{Ne}^{9+}$ & $3 / 2$ & -0.661797 & $9.3502 \times 10^{-04}$ & $-4.0386 \times 10^{-07}$ & $1.7379 \times 10^{-07}$ & $-3.4625 \times 10^{-07}$ & $9.3444 \times 10^{-04}$ & $1.3268 \times 10^{+06}$ \\
\hline${ }^{23} \mathrm{Na}^{10+}$ & $3 / 2$ & 2.217656 & $4.1771 \times 10^{-03}$ & $-1.9480 \times 10^{-06}$ & $8.5200 \times 10^{-07}$ & $-2.1893 \times 10^{-06}$ & $4.1738 \times 10^{-03}$ & $2.9705 \times 10^{+05}$ \\
\hline${ }^{25} \mathrm{Mg}^{11+}$ & $5 / 2$ & -0.85545 & $1.8859 \times 10^{-03}$ & $-9.9815 \times 10^{-07}$ & $4.1870 \times 10^{-07}$ & $-1.2774 \times 10^{-06}$ & $1.8841 \times 10^{-03}$ & $6.5807 \times 10^{+05}$ \\
\hline${ }^{27} \mathrm{Al}^{12+}$ & $5 / 2$ & 3.641507 & $1.0227 \times 10^{-02}$ & $-5.8177 \times 10^{-06}$ & $2.4545 \times 10^{-06}$ & $-8.4874 \times 10^{-06}$ & $1.0215 \times 10^{-02}$ & $1.2138 \times 10^{+05}$ \\
\hline${ }^{29} \mathrm{Si}^{13+}$ & $1 / 2$ & -0.55529 & $3.2528 \times 10^{-03}$ & $-2.0701 \times 10^{-06}$ & $8.3909 \times 10^{-07}$ & $-3.1939 \times 10^{-06}$ & $3.2484 \times 10^{-03}$ & $3.8168 \times 10^{+05}$ \\
\hline${ }^{31} \mathrm{P}^{14+}$ & $1 / 2$ & 1.1316 & $8.1710 \times 10^{-03}$ & $-5.7216 \times 10^{-06}$ & $2.2542 \times 10^{-06}$ & $-9.2596 \times 10^{-06}$ & $8.1583 \times 10^{-03}$ & $1.5197 \times 10^{+05}$ \\
\hline${ }^{33} \mathrm{~S}^{15+}$ & $3 / 2$ & 0.643821 & $3.7702 \times 10^{-03}$ & $-2.9070 \times 10^{-06}$ & $1.1076 \times 10^{-06}$ & $-4.8407 \times 10^{-06}$ & $3.7636 \times 10^{-03}$ & $3.2943 \times 10^{+05}$ \\
\hline${ }^{35} \mathrm{Cl}^{16+}$ & $3 / 2$ & 0.821874 & $5.7874 \times 10^{-03}$ & $-4.8693 \times 10^{-06}$ & $1.8037 \times 10^{-06}$ & $-8.2993 \times 10^{-06}$ & $5.7760 \times 10^{-03}$ & $2.1465 \times 10^{+05}$ \\
\hline${ }^{37} \mathrm{Cl}^{16+}$ & $3 / 2$ & 0.684124 & $4.8172 \times 10^{-03}$ & $-4.1141 \times 10^{-06}$ & $1.5014 \times 10^{-06}$ & $-6.9080 \times 10^{-06}$ & $4.8077 \times 10^{-03}$ & $2.5789 \times 10^{+05}$ \\
\hline${ }^{39} \mathrm{~K}^{18+}$ & $3 / 2$ & 0.391507 & $3.8702 \times 10^{-03}$ & $-3.8177 \times 10^{-06}$ & $1.3446 \times 10^{-06}$ & $-6.7049 \times 10^{-06}$ & $3.8610 \times 10^{-03}$ & $3.2112 \times 10^{+05}$ \\
\hline${ }^{41} \mathrm{~K}^{18+}$ & $3 / 2$ & 0.214893 & $2.1242 \times 10^{-03}$ & $-2.1238 \times 10^{-06}$ & $7.3798 \times 10^{-07}$ & $-3.6801 \times 10^{-06}$ & $2.1192 \times 10^{-03}$ & $5.8507 \times 10^{+05}$ \\
\hline${ }^{43} \mathrm{Ca}^{19+}$ & $7 / 2$ & -1.317643 & $1.3060 \times 10^{-02}$ & $-1.4145 \times 10^{-05}$ & $4.7709 \times 10^{-06}$ & $-2.4564 \times 10^{-05}$ & $1.3026 \times 10^{-02}$ & $9.5179 \times 10^{+04}$ \\
\hline${ }^{45} \mathrm{Sc}^{20+}$ & $7 / 2$ & 4.756487 & $5.4753 \times 10^{-02}$ & $-6.2716 \times 10^{-05}$ & $2.0981 \times 10^{-05}$ & $-1.1108 \times 10^{-04}$ & $5.4601 \times 10^{-02}$ & $2.2708 \times 10^{+04}$ \\
\hline${ }^{47} \mathrm{Ti}^{21+}$ & $5 / 2$ & -0.78848 & $1.0994 \times 10^{-02}$ & $-1.3463 \times 10^{-05}$ & $4.4099 \times 10^{-06}$ & $-2.3924 \times 10^{-05}$ & $1.0961 \times 10^{-02}$ & $1.1311 \times 10^{+05}$ \\
\hline${ }^{49} \mathrm{Ti}^{21+}$ & $7 / 2$ & -1.10417 & $1.4662 \times 10^{-02}$ & $-1.8160 \times 10^{-05}$ & $5.8813 \times 10^{-06}$ & $-3.1906 \times 10^{-05}$ & $1.4618 \times 10^{-02}$ & $8.4814 \times 10^{+04}$ \\
\hline${ }^{51} \mathrm{~V}^{22+}$ & $7 / 2$ & 5.148706 & $7.8403 \times 10^{-02}$ & $-1.0213 \times 10^{-04}$ & $3.2855 \times 10^{-05}$ & $-1.8212 \times 10^{-04}$ & $7.8152 \times 10^{-02}$ & $1.5865 \times 10^{+04}$ \\
\hline${ }^{53} \mathrm{Cr}^{23+}$ & $3 / 2$ & -0.47454 & $9.6136 \times 10^{-03}$ & $-1.3474 \times 10^{-05}$ & $4.2015 \times 10^{-06}$ & $-2.3739 \times 10^{-05}$ & $9.5806 \times 10^{-03}$ & $1.2941 \times 10^{+05}$ \\
\hline${ }^{55} \mathrm{Mn}^{24+}$ & $5 / 2$ & 3.468719 & $7.1761 \times 10^{-02}$ & $-1.0682 \times 10^{-04}$ & $3.2656 \times 10^{-05}$ & $-1.8768 \times 10^{-04}$ & $7.1499 \times 10^{-02}$ & $1.7341 \times 10^{+04}$ \\
\hline${ }^{57} \mathrm{Fe}^{25+}$ & $1 / 2$ & 0.090623 & $3.5289 \times 10^{-03}$ & $-5.6000 \times 10^{-06}$ & $1.6697 \times 10^{-06}$ & $-9.7435 \times 10^{-06}$ & $3.5152 \times 10^{-03}$ & $3.5270 \times 10^{+05}$ \\
\hline${ }^{59} \mathrm{Co}^{26+}$ & $7 / 2$ & 4.627 & $1.1579 \times 10^{-01}$ & $-1.9449 \times 10^{-04}$ & $5.6884 \times 10^{-05}$ & $-3.3652 \times 10^{-04}$ & $1.1531 \times 10^{-01}$ & $1.0752 \times 10^{+04}$ \\
\hline${ }^{61} \mathrm{Ni}^{27+}$ & $3 / 2$ & -0.75002 & $2.4527 \times 10^{-02}$ & $-4.3927 \times 10^{-05}$ & $1.2496 \times 10^{-05}$ & $-7.4842 \times 10^{-05}$ & $2.4421 \times 10^{-02}$ & $5.0770 \times 10^{+04}$ \\
\hline${ }^{63} \mathrm{Cu}^{28+}$ & $3 / 2$ & 2.227346 & $8.1290 \times 10^{-02}$ & $-1.5482 \times 10^{-04}$ & $4.2900 \times 10^{-05}$ & $-2.5982 \times 10^{-04}$ & $8.0918 \times 10^{-02}$ & $1.5322 \times 10^{+04}$ \\
\hline${ }^{65} \mathrm{Cu}^{28+}$ & $3 / 2$ & 2.38167 & $8.6918 \times 10^{-02}$ & $-1.6695 \times 10^{-04}$ & $4.5871 \times 10^{-05}$ & $-2.7781 \times 10^{-04}$ & $8.6519 \times 10^{-02}$ & $1.4330 \times 10^{+04}$ \\
\hline${ }^{67} \mathrm{Zn}^{29+}$ & $5 / 2$ & 0.875479 & $3.1982 \times 10^{-02}$ & $-6.5528 \times 10^{-05}$ & $1.7465 \times 10^{-05}$ & $-1.0685 \times 10^{-04}$ & $3.1827 \times 10^{-02}$ & $3.8956 \times 10^{+04}$ \\
\hline${ }^{69} \mathrm{Ga}^{30+}$ & $3 / 2$ & 2.016589 & $9.0762 \times 10^{-02}$ & $-1.9436 \times 10^{-04}$ & $5.1238 \times 10^{-05}$ & $-3.1632 \times 10^{-04}$ & $9.0303 \times 10^{-02}$ & $1.3730 \times 10^{+04}$ \\
\hline${ }^{71} \mathrm{Ga}^{30+}$ & $3 / 2$ & 2.562266 & $1.1532 \times 10^{-01}$ & $-2.4886 \times 10^{-04}$ & $6.5099 \times 10^{-05}$ & $-4.0190 \times 10^{-04}$ & $1.1473 \times 10^{-01}$ & $1.0807 \times 10^{+04}$ \\
\hline${ }^{73} \mathrm{Ge}^{31+}$ & $9 / 2$ & -0.879468 & $3.6464 \times 10^{-02}$ & $-8.3276 \times 10^{-05}$ & $2.1260 \times 10^{-05}$ & $-1.3234 \times 10^{-04}$ & $3.6269 \times 10^{-02}$ & $3.4184 \times 10^{+04}$ \\
\hline${ }^{75} \mathrm{As}^{32+}$ & $3 / 2$ & 1.439475 & $7.8959 \times 10^{-02}$ & $-1.8967 \times 10^{-04}$ & $4.7507 \times 10^{-05}$ & $-2.9795 \times 10^{-04}$ & $7.8518 \times 10^{-02}$ & $1.5790 \times 10^{+04}$ \\
\hline${ }^{77} \mathrm{Se}^{33+}$ & $1 / 2$ & 0.535042 & $4.8410 \times 10^{-02}$ & $-1.2213 \times 10^{-04}$ & $3.0033 \times 10^{-05}$ & $-1.8965 \times 10^{-04}$ & $4.8128 \times 10^{-02}$ & $2.5761 \times 10^{+04}$ \\
\hline${ }^{79} \mathrm{Br}^{34+}$ & $3 / 2$ & 2.1064 & $1.3938 \times 10^{-01}$ & $-3.6985 \times 10^{-04}$ & $8.9097 \times 10^{-05}$ & $-5.6612 \times 10^{-04}$ & $1.3853 \times 10^{-01}$ & $8.9497 \times 10^{+03}$ \\
\hline${ }^{81} \mathrm{Br}^{34+}$ & $3 / 2$ & 2.270562 & $1.5024 \times 10^{-01}$ & $-4.0133 \times 10^{-04}$ & $9.6035 \times 10^{-05}$ & $-6.1021 \times 10^{-04}$ & $1.4932 \times 10^{-01}$ & $8.3033 \times 10^{+03}$ \\
\hline${ }^{83} \mathrm{Kr}^{35+}$ & $9 / 2$ & -0.970669 & $5.8585 \times 10^{-02}$ & $-1.6307 \times 10^{-04}$ & $3.8560 \times 10^{-05}$ & $-2.4639 \times 10^{-04}$ & $5.8214 \times 10^{-02}$ & $2.1298 \times 10^{+04}$ \\
\hline${ }^{85} \mathrm{Rb}^{36+}$ & $5 / 2$ & 1.357 & $9.6601 \times 10^{-02}$ & $-2.8479 \times 10^{-04}$ & $6.5424 \times 10^{-05}$ & $-4.2020 \times 10^{-04}$ & $9.5962 \times 10^{-02}$ & $1.2920 \times 10^{+04}$ \\
\hline${ }^{87} \mathrm{Sr}^{37+}$ & $9 / 2$ & -1.093603 & $7.8586 \times 10^{-02}$ & $-2.3874 \times 10^{-04}$ & $5.4733 \times 10^{-05}$ & $-3.5317 \times 10^{-04}$ & $7.8049 \times 10^{-02}$ & $1.5885 \times 10^{+04}$ \\
\hline${ }^{89} \mathrm{Y}^{38+}$ & $1 / 2$ & -0.137415 & $1.9337 \times 10^{-02}$ & $-6.1982 \times 10^{-05}$ & $1.3841 \times 10^{-05}$ & $-8.9692 \times 10^{-05}$ & $1.9199 \times 10^{-02}$ & $6.4578 \times 10^{+04}$ \\
\hline${ }^{91} \mathrm{Zr}^{39+}$ & $5 / 2$ & -1.30362 & $1.1953 \times 10^{-01}$ & $-4.0303 \times 10^{-04}$ & $8.7889 \times 10^{-05}$ & $-5.7171 \times 10^{-04}$ & $1.1864 \times 10^{-01}$ & $1.0450 \times 10^{+04}$ \\
\hline${ }^{93} \mathrm{Nb}^{40+}$ & $9 / 2$ & 6.1705 & $5.6799 \times 10^{-01}$ & $-2.0083 \times 10^{-03}$ & $4.2878 \times 10^{-04}$ & $-2.7988 \times 10^{-03}$ & $5.6361 \times 10^{-01}$ & $2.1998 \times 10^{+03}$ \\
\hline${ }^{95} \mathrm{Mo}^{41+}$ & $5 / 2$ & -0.9142 & $9.8377 \times 10^{-02}$ & $-3.6551 \times 10^{-04}$ & $7.6211 \times 10^{-05}$ & $-4.9903 \times 10^{-04}$ & $9.7588 \times 10^{-02}$ & $1.2705 \times 10^{+04}$ \\
\hline${ }^{97} \mathrm{Mo}^{41+}$ & $5 / 2$ & -0.9335 & $1.0045 \times 10^{-01}$ & $-3.7525 \times 10^{-04}$ & $7.7814 \times 10^{-05}$ & $-5.0953 \times 10^{-04}$ & $9.9640 \times 10^{-02}$ & $1.2443 \times 10^{+04}$ \\
\hline${ }^{99} \mathrm{Tc}^{42+}$ & $9 / 2$ & 5.6847 & $6.1214 \times 10^{-01}$ & $-2.4050 \times 10^{-03}$ & $4.8642 \times 10^{-04}$ & $-3.1942 \times 10^{-03}$ & $6.0703 \times 10^{-01}$ & $2.0425 \times 10^{+03}$ \\
\hline${ }^{101} \mathrm{Ru}^{43+}$ & $5 / 2$ & -0.7188 & $9.0227 \times 10^{-02}$ & $-3.7096 \times 10^{-04}$ & $7.3509 \times 10^{-05}$ & $-4.8395 \times 10^{-04}$ & $8.9445 \times 10^{-02}$ & $1.3861 \times 10^{+04}$ \\
\hline${ }^{99} \mathrm{Ru}^{43+}$ & $5 / 2$ & -0.6413 & $8.0504 \times 10^{-02}$ & $-3.2928 \times 10^{-04}$ & $6.5588 \times 10^{-05}$ & $-4.3180 \times 10^{-04}$ & $7.9809 \times 10^{-02}$ & $1.5535 \times 10^{+04}$ \\
\hline${ }^{103} \mathrm{Rh}^{44+}$ & $1 / 2$ & -0.0884 & $1.9934 \times 10^{-02}$ & $-8.5945 \times 10^{-05}$ & $1.6644 \times 10^{-05}$ & $-1.0983 \times 10^{-04}$ & $1.9755 \times 10^{-02}$ & $6.2762 \times 10^{+04}$ \\
\hline${ }^{105} \mathrm{Pd}^{45+}$ & $5 / 2$ & -0.642 & $9.3538 \times 10^{-02}$ & $-4.2482 \times 10^{-04}$ & $8.0015 \times 10^{-05}$ & $-5.2908 \times 10^{-04}$ & $9.2664 \times 10^{-02}$ & $1.3380 \times 10^{+04}$ \\
\hline${ }^{107} \mathrm{Ag}^{46+}$ & $1 / 2$ & -0.11368 & $2.9678 \times 10^{-02}$ & $-1.4203 \times 10^{-04}$ & $2.5999 \times 10^{-05}$ & $-1.7223 \times 10^{-04}$ & $2.9390 \times 10^{-02}$ & $4.2187 \times 10^{+04}$ \\
\hline${ }^{109} \mathrm{Ag}^{46+}$ & $1 / 2$ & -0.130691 & $3.4116 \times 10^{-02}$ & $-1.6402 \times 10^{-04}$ & $2.9887 \times 10^{-05}$ & $-1.9799 \times 10^{-04}$ & $3.3784 \times 10^{-02}$ & $3.6699 \times 10^{+04}$ \\
\hline${ }^{111} \mathrm{Cd}^{47+}$ & $1 / 2$ & -0.594886 & $1.6679 \times 10^{-01}$ & $-8.3681 \times 10^{-04}$ & $1.4958 \times 10^{-04}$ & $-9.9253 \times 10^{-04}$ & $1.6511 \times 10^{-01}$ & $7.5093 \times 10^{+03}$ \\
\hline${ }^{113} \mathrm{Cd}^{47+}$ & $1 / 2$ & -0.622301 & $1.7446 \times 10^{-01}$ & $-8.7915 \times 10^{-04}$ & $1.5647 \times 10^{-04}$ & $-1.0382 \times 10^{-03}$ & $1.7270 \times 10^{-01}$ & $7.1792 \times 10^{+03}$ \\
\hline
\end{tabular}

Table 5. Hyperfine transition energy $\Delta E_{H}$ in $[\mathrm{eV}]$ and in wavelenghts $\lambda_{H}[\mathrm{~nm}]$. 


\begin{tabular}{|c|c|c|c|c|c|c|c|c|}
\hline Label & $I$ & $\mu_{I}$ & $\Delta E_{H}^{H f s}$ & $\Delta E_{H}^{B W}$ & $V P_{H}^{h f s}$ & $S E_{H}$ & $\Delta E_{H}$ & $\lambda_{H}$ \\
\hline${ }^{113} \operatorname{In}^{48+}$ & $9 / 2$ & 5.5289 & $9.2392 \times 10^{-01}$ & $-4.8403 \times 10^{-03}$ & $8.4802 \times 10^{-04}$ & $-5.6350 \times 10^{-03}$ & $9.1429 \times 10^{-01}$ & $1.3561 \times 10^{+03}$ \\
\hline${ }^{115} \operatorname{In}^{48+}$ & $9 / 2$ & 5.5408 & $9.2584 \times 10^{-01}$ & $-4.8712 \times 10^{-03}$ & $8.4978 \times 10^{-04}$ & $-5.6467 \times 10^{-03}$ & $9.1617 \times 10^{-01}$ & $1.3533 \times 10^{+03}$ \\
\hline${ }^{115} \mathrm{Sn}^{49+}$ & $1 / 2$ & -0.91883 & $2.9625 \times 10^{-01}$ & $-1.6081 \times 10^{-03}$ & $2.7819 \times 10^{-04}$ & $-1.8509 \times 10^{-03}$ & $2.9307 \times 10^{-01}$ & $4.2305 \times 10^{+03}$ \\
\hline${ }^{117} \mathrm{Sn}^{49+}$ & $1 / 2$ & -1.00104 & $3.2273 \times 10^{-01}$ & $-1.7592 \times 10^{-03}$ & $3.0306 \times 10^{-04}$ & $-2.0163 \times 10^{-03}$ & $3.1926 \times 10^{-01}$ & $3.8835 \times 10^{+03}$ \\
\hline${ }^{119} \mathrm{Sn}^{49+}$ & $1 / 2$ & -1.04728 & $3.3761 \times 10^{-01}$ & $-1.8480 \times 10^{-03}$ & $3.1703 \times 10^{-04}$ & $-2.1093 \times 10^{-03}$ & $3.3397 \times 10^{-01}$ & $3.7124 \times 10^{+03}$ \\
\hline${ }^{121} \mathrm{Sb}^{50+}$ & $5 / 2$ & 3.3634 & $6.9645 \times 10^{-01}$ & $-4.0068 \times 10^{-03}$ & $6.6889 \times 10^{-04}$ & $-4.4553 \times 10^{-03}$ & $6.8866 \times 10^{-01}$ & $1.8004 \times 10^{+03}$ \\
\hline${ }^{123} \mathrm{Sb}^{50+}$ & $7 / 2$ & 2.5498 & $5.0280 \times 10^{-01}$ & $-2.9042 \times 10^{-03}$ & $4.8290 \times 10^{-04}$ & $-3.2165 \times 10^{-03}$ & $4.9716 \times 10^{-01}$ & $2.4939 \times 10^{+03}$ \\
\hline${ }^{123} \mathrm{Te}^{51+}$ & $1 / 2$ & -0.736948 & $2.7206 \times 10^{-01}$ & $-1.6328 \times 10^{-03}$ & $2.6717 \times 10^{-04}$ & $-1.7813 \times 10^{-03}$ & $2.6891 \times 10^{-01}$ & $4.6106 \times 10^{+03}$ \\
\hline${ }^{125} \mathrm{Te}^{51+}$ & $1 / 2$ & -0.888505 & $3.2798 \times 10^{-01}$ & $-1.9761 \times 10^{-03}$ & $3.2209 \times 10^{-04}$ & $-2.1474 \times 10^{-03}$ & $3.2418 \times 10^{-01}$ & $3.8245 \times 10^{+03}$ \\
\hline${ }^{127} \mathrm{I}^{52+}$ & $5 / 2$ & 2.813273 & $6.6600 \times 10^{-01}$ & $-4.1711 \times 10^{-03}$ & $6.6855 \times 10^{-04}$ & $-4.4611 \times 10^{-03}$ & $6.5804 \times 10^{-01}$ & $1.8842 \times 10^{+03}$ \\
\hline${ }^{129} \mathrm{Xe}^{53+}$ & $1 / 2$ & -0.777976 & $3.2783 \times 10^{-01}$ & $-2.1320 \times 10^{-03}$ & $3.3631 \times 10^{-04}$ & $-2.2457 \times 10^{-03}$ & $3.2379 \times 10^{-01}$ & $3.8291 \times 10^{+03}$ \\
\hline${ }^{131} \mathrm{Xe}^{53+}$ & $3 / 2$ & 0.691862 & $1.9434 \times 10^{-01}$ & $-1.2686 \times 10^{-03}$ & $1.9937 \times 10^{-04}$ & $-1.3313 \times 10^{-03}$ & $1.9194 \times 10^{-01}$ & $6.4594 \times 10^{+03}$ \\
\hline${ }^{133} \mathrm{Cs}^{54+}$ & $7 / 2$ & 2.582025 & $6.6335 \times 10^{-01}$ & $-4.5193 \times 10^{-03}$ & $6.9525 \times 10^{-04}$ & $-4.6453 \times 10^{-03}$ & $6.5488 \times 10^{-01}$ & $1.8932 \times 10^{+03}$ \\
\hline${ }^{135} \mathrm{Ba}^{55+}$ & $3 / 2$ & 0.837943 & $2.6782 \times 10^{-01}$ & $-1.8959 \times 10^{-03}$ & $2.8671 \times 10^{-04}$ & $-1.9166 \times 10^{-03}$ & $2.6429 \times 10^{-01}$ & $4.6912 \times 10^{+03}$ \\
\hline${ }^{137} \mathrm{Ba}^{55+}$ & $3 / 2$ & 0.937365 & $2.9957 \times 10^{-01}$ & $-2.1281 \times 10^{-03}$ & $3.2070 \times 10^{-04}$ & $-2.1438 \times 10^{-03}$ & $2.9562 \times 10^{-01}$ & $4.1941 \times 10^{+03}$ \\
\hline${ }^{138} \mathrm{La}^{56+}$ & 5 & 3.713646 & $1.0432 \times 10^{+00}$ & $-7.7300 \times 10^{-03}$ & $1.1405 \times 10^{-03}$ & $-7.6271 \times 10^{-03}$ & $1.0290 \times 10^{+00}$ & $1.2049 \times 10^{+03}$ \\
\hline${ }^{139} \mathrm{La}^{56+}$ & $7 / 2$ & 2.783046 & $8.1223 \times 10^{-01}$ & $-6.0286 \times 10^{-03}$ & $8.8797 \times 10^{-04}$ & $-5.9382 \times 10^{-03}$ & $8.0115 \times 10^{-01}$ & $1.5476 \times 10^{+03}$ \\
\hline${ }^{141} \operatorname{Pr}^{58+}$ & $5 / 2$ & 4.2754 & $1.4846 \times 10^{+00}$ & $-1.1941 \times 10^{-02}$ & $1.6914 \times 10^{-03}$ & $-1.1317 \times 10^{-02}$ & $1.4630 \times 10^{+00}$ & $8.4747 \times 10^{+02}$ \\
\hline${ }^{143} \mathrm{Nd}^{59+}$ & $7 / 2$ & -1.065 & $3.7453 \times 10^{-01}$ & $-3.1431 \times 10^{-03}$ & $4.3547 \times 10^{-04}$ & $-2.9143 \times 10^{-03}$ & $3.6891 \times 10^{-01}$ & $3.3609 \times 10^{+03}$ \\
\hline${ }^{145} \mathrm{Nd}^{59+}$ & $7 / 2$ & -0.656 & $2.3067 \times 10^{-01}$ & $-1.9420 \times 10^{-03}$ & $2.6820 \times 10^{-04}$ & $-1.7949 \times 10^{-03}$ & $2.2720 \times 10^{-01}$ & $5.4570 \times 10^{+03}$ \\
\hline${ }^{147} \mathrm{Sm}^{61+}$ & $7 / 2$ & -0.8148 & $3.2340 \times 10^{-01}$ & $-2.9753 \times 10^{-03}$ & $3.9139 \times 10^{-04}$ & $-2.6197 \times 10^{-03}$ & $3.1819 \times 10^{-01}$ & $3.8965 \times 10^{+03}$ \\
\hline${ }^{149} \mathrm{Sm}^{61+}$ & $7 / 2$ & -0.6708 & $2.6621 \times 10^{-01}$ & $-2.4566 \times 10^{-03}$ & $3.2218 \times 10^{-04}$ & $-2.1565 \times 10^{-03}$ & $2.6192 \times 10^{-01}$ & $4.7337 \times 10^{+03}$ \\
\hline${ }^{151} \mathrm{Eu}^{62+}$ & $5 / 2$ & 3.4717 & $1.5358 \times 10^{+00}$ & $-1.4803 \times 10^{-02}$ & $1.8958 \times 10^{-03}$ & $-1.2689 \times 10^{-02}$ & $1.5102 \times 10^{+00}$ & $8.2100 \times 10^{+02}$ \\
\hline${ }^{153} \mathrm{Eu}^{62+}$ & $5 / 2$ & 1.533 & $6.7807 \times 10^{-01}$ & $-6.5548 \times 10^{-03}$ & $8.3701 \times 10^{-04}$ & $-5.6025 \times 10^{-03}$ & $6.6675 \times 10^{-01}$ & $1.8595 \times 10^{+03}$ \\
\hline${ }^{155} \mathrm{Gd}^{63+}$ & $3 / 2$ & -0.33726 & $1.7591 \times 10^{-01}$ & $-1.7666 \times 10^{-03}$ & $2.2144 \times 10^{-04}$ & $-1.4822 \times 10^{-03}$ & $1.7289 \times 10^{-01}$ & $7.1715 \times 10^{+03}$ \\
\hline${ }^{155} \mathrm{Gd}^{63+}$ & $3 / 2$ & -0.33726 & $1.7591 \times 10^{-01}$ & $-1.7666 \times 10^{-03}$ & $2.2144 \times 10^{-04}$ & $-1.4822 \times 10^{-03}$ & $1.7289 \times 10^{-01}$ & $7.1715 \times 10^{+03}$ \\
\hline${ }^{159} \mathrm{~Tb}^{64+}$ & $3 / 2$ & 2.014 & $1.1147 \times 10^{+00}$ & $-1.1553 \times 10^{-02}$ & $1.4307 \times 10^{-03}$ & $-9.5756 \times 10^{-03}$ & $1.0950 \times 10^{+00}$ & $1.1322 \times 10^{+03}$ \\
\hline${ }^{161} \mathrm{Dy}^{65+}$ & $5 / 2$ & -0.4803 & $2.5355 \times 10^{-01}$ & $-2.7673 \times 10^{-03}$ & $3.3174 \times 10^{-04}$ & $-2.2201 \times 10^{-03}$ & $2.4889 \times 10^{-01}$ & $4.9814 \times 10^{+03}$ \\
\hline${ }^{163} \mathrm{Dy}^{65+}$ & $5 / 2$ & 0.6726 & $3.5502 \times 10^{-01}$ & $-3.8850 \times 10^{-03}$ & $4.6450 \times 10^{-04}$ & $-3.1086 \times 10^{-03}$ & $3.4849 \times 10^{-01}$ & $3.5578 \times 10^{+03}$ \\
\hline${ }^{165} \mathrm{Ho}^{66+}$ & $7 / 2$ & 4.132 & $2.2019 \times 10^{+00}$ & $-2.4978 \times 10^{-02}$ & $2.9364 \times 10^{-03}$ & $-1.9649 \times 10^{-02}$ & $2.1602 \times 10^{+00}$ & $5.7394 \times 10^{+02}$ \\
\hline${ }^{165} \mathrm{Ho}^{66+}$ & $7 / 2$ & 4.173 & $2.2238 \times 10^{+00}$ & $-2.5226 \times 10^{-02}$ & $2.9656 \times 10^{-03}$ & $-1.9844 \times 10^{-02}$ & $2.1817 \times 10^{+00}$ & $5.6830 \times 10^{+02}$ \\
\hline${ }^{167} \mathrm{Er}^{67+}$ & $7 / 2$ & -0.56385 & $3.1828 \times 10^{-01}$ & $-3.7712 \times 10^{-03}$ & $4.3256 \times 10^{-04}$ & $-2.8941 \times 10^{-03}$ & $3.1205 \times 10^{-01}$ & $3.9732 \times 10^{+03}$ \\
\hline${ }^{169} \mathrm{Tm}^{68+}$ & $1 / 2$ & -0.2316 & $2.4240 \times 10^{-01}$ & $-2.9679 \times 10^{-03}$ & $3.3567 \times 10^{-04}$ & $-2.2456 \times 10^{-03}$ & $2.3752 \times 10^{-01}$ & $5.2199 \times 10^{+03}$ \\
\hline${ }^{171} \mathrm{Yb}^{69+}$ & $1 / 2$ & 0.49367 & $5.4692 \times 10^{-01}$ & $-6.9993 \times 10^{-03}$ & $7.7158 \times 10^{-04}$ & $-5.1610 \times 10^{-03}$ & $5.3553 \times 10^{-01}$ & $2.3152 \times 10^{+03}$ \\
\hline${ }^{173} \mathrm{Yb}^{69+}$ & $5 / 2$ & -0.67989 & $4.5187 \times 10^{-01}$ & $-5.7968 \times 10^{-03}$ & $6.3749 \times 10^{-04}$ & $-4.2641 \times 10^{-03}$ & $4.4244 \times 10^{-01}$ & $2.8023 \times 10^{+03}$ \\
\hline${ }^{175} \mathrm{Lu}^{70+}$ & $7 / 2$ & 2.2323 & $1.4949 \times 10^{+00}$ & $-2.0097 \times 10^{-02}$ & $2.1482 \times 10^{-03}$ & $-1.4367 \times 10^{-02}$ & $1.4625 \times 10^{+00}$ & $8.4773 \times 10^{+02}$ \\
\hline${ }^{176} \mathrm{Lu}^{70+}$ & 7 & 3.1692 & $1.9895 \times 10^{+00}$ & $-2.6778 \times 10^{-02}$ & $2.8590 \times 10^{-03}$ & $-1.9121 \times 10^{-02}$ & $1.9464 \times 10^{+00}$ & $6.3699 \times 10^{+02}$ \\
\hline${ }^{177} \mathrm{Hf}^{71+}$ & $7 / 2$ & 0.7935 & $5.6266 \times 10^{-01}$ & $-7.7861 \times 10^{-03}$ & $8.2353 \times 10^{-04}$ & $-5.5070 \times 10^{-03}$ & $5.5019 \times 10^{-01}$ & $2.2535 \times 10^{+03}$ \\
\hline${ }^{179} \mathrm{Hf}^{71+}$ & $9 / 2$ & -0.6409 & $4.4176 \times 10^{-01}$ & $-6.1271 \times 10^{-03}$ & $6.4658 \times 10^{-04}$ & $-4.3237 \times 10^{-03}$ & $4.3195 \times 10^{-01}$ & $2.8703 \times 10^{+03}$ \\
\hline${ }^{180} \mathrm{Ta}^{72+}$ & 9 & 4.77 & $3.3064 \times 10^{+00}$ & $-4.7281 \times 10^{-02}$ & $4.9282 \times 10^{-03}$ & $-3.2951 \times 10^{-02}$ & $3.2311 \times 10^{+00}$ & $3.8372 \times 10^{+02}$ \\
\hline${ }^{181} \mathrm{Ta}^{72+}$ & $7 / 2$ & 2.3705 & $1.7789 \times 10^{+00}$ & $-2.5467 \times 10^{-02}$ & $2.6514 \times 10^{-03}$ & $-1.7728 \times 10^{-02}$ & $1.7383 \times 10^{+00}$ & $7.1323 \times 10^{+02}$ \\
\hline${ }^{183} \mathrm{~W}^{73+}$ & $1 / 2$ & 0.117785 & $1.6354 \times 10^{-01}$ & $-2.4482 \times 10^{-03}$ & $2.4819 \times 10^{-04}$ & $-1.6593 \times 10^{-03}$ & $1.5968 \times 10^{-01}$ & $7.7647 \times 10^{+03}$ \\
\hline${ }^{185} \mathrm{Re}^{74+}$ & $5 / 2$ & 3.1871 & $2.8090 \times 10^{+00}$ & $-4.3454 \times 10^{-02}$ & $4.3401 \times 10^{-03}$ & $-2.9014 \times 10^{-02}$ & $2.7408 \times 10^{+00}$ & $4.5236 \times 10^{+02}$ \\
\hline${ }^{187} \operatorname{Re}^{74+}$ & $5 / 2$ & 3.2197 & $2.8372 \times 10^{+00}$ & $-4.3986 \times 10^{-02}$ & $4.3838 \times 10^{-03}$ & $-2.9305 \times 10^{-02}$ & $2.7683 \times 10^{+00}$ & $4.4787 \times 10^{+02}$ \\
\hline${ }^{187} \mathrm{Os}^{75+}$ & $1 / 2$ & 0.064652 & $1.0039 \times 10^{-01}$ & $-1.6185 \times 10^{-03}$ & $1.5789 \times 10^{-04}$ & $-1.0555 \times 10^{-03}$ & $9.7873 \times 10^{-02}$ & $1.2668 \times 10^{+04}$ \\
\hline${ }^{189} \mathrm{Os}^{75+}$ & $3 / 2$ & 0.659933 & $6.8302 \times 10^{-01}$ & $-1.1035 \times 10^{-02}$ & $1.0743 \times 10^{-03}$ & $-7.1811 \times 10^{-03}$ & $6.6588 \times 10^{-01}$ & $1.8620 \times 10^{+03}$ \\
\hline${ }^{191} \operatorname{Ir} 76+$ & $3 / 2$ & 0.1507 & $1.6492 \times 10^{-01}$ & $-2.7614 \times 10^{-03}$ & $2.6402 \times 10^{-04}$ & $-1.7648 \times 10^{-03}$ & $1.6066 \times 10^{-01}$ & $7.7171 \times 10^{+03}$ \\
\hline${ }^{193} \operatorname{Ir}^{76+}$ & $3 / 2$ & 0.1637 & $1.7912 \times 10^{-01}$ & $-3.0051 \times 10^{-03}$ & $2.8674 \times 10^{-04}$ & $-1.9167 \times 10^{-03}$ & $1.7448 \times 10^{-01}$ & $7.1058 \times 10^{+03}$ \\
\hline${ }^{195} \mathrm{Pt}^{77+}$ & $1 / 2$ & 0.60952 & $1.0579 \times 10^{+00}$ & $-1.8348 \times 10^{-02}$ & $1.7235 \times 10^{-03}$ & $-1.1521 \times 10^{-02}$ & $1.0298 \times 10^{+00}$ & $1.2040 \times 10^{+03}$ \\
\hline${ }^{197} \mathrm{Au}^{78+}$ & $3 / 2$ & 0.148158 & $1.8109 \times 10^{-01}$ & $-3.2807 \times 10^{-03}$ & $3.0021 \times 10^{-04}$ & $-2.0068 \times 10^{-03}$ & $1.7610 \times 10^{-01}$ & $7.0405 \times 10^{+03}$ \\
\hline${ }^{199} \mathrm{Hg}^{79+}$ & $1 / 2$ & 0.505885 & $9.8030 \times 10^{-01}$ & $-1.8419 \times 10^{-02}$ & $1.6535 \times 10^{-03}$ & $-1.1054 \times 10^{-02}$ & $9.5248 \times 10^{-01}$ & $1.3017 \times 10^{+03}$ \\
\hline${ }^{201} \mathrm{Hg}^{79+}$ & $3 / 2$ & -0.560226 & $7.2359 \times 10^{-01}$ & $-1.3621 \times 10^{-02}$ & $1.2205 \times 10^{-03}$ & $-8.1591 \times 10^{-03}$ & $7.0303 \times 10^{-01}$ & $1.7636 \times 10^{+03}$ \\
\hline${ }^{203} \mathrm{Tl}^{80+}$ & $1 / 2$ & 1.622258 & $3.3212 \times 10^{+00}$ & $-6.4910 \times 10^{-02}$ & $5.6992 \times 10^{-03}$ & $-3.8104 \times 10^{-02}$ & $3.2239 \times 10^{+00}$ & $3.8457 \times 10^{+02}$ \\
\hline${ }^{205} \mathrm{Tl}^{80+}$ & $1 / 2$ & 1.638215 & $3.3532 \times 10^{+00}$ & $-6.5655 \times 10^{-02}$ & $5.7540 \times 10^{-03}$ & $-3.8471 \times 10^{-02}$ & $3.2549 \times 10^{+00}$ & $3.8092 \times 10^{+02}$ \\
\hline${ }^{207} \mathrm{~Pb}^{81+}$ & $1 / 2$ & 0.58219 & $1.2589 \times 10^{+00}$ & $-2.5634 \times 10^{-02}$ & $2.1975 \times 10^{-03}$ & $-1.4694 \times 10^{-02}$ & $1.2208 \times 10^{+00}$ & $1.0156 \times 10^{+03}$ \\
\hline${ }^{207} \mathrm{~Pb}^{81+}$ & $1 / 2$ & 0.592583 & $1.2815 \times 10^{+00}$ & $-2.6071 \times 10^{-02}$ & $2.2369 \times 10^{-03}$ & $-1.4958 \times 10^{-02}$ & $1.2427 \times 10^{+00}$ & $9.9767 \times 10^{+02}$ \\
\hline${ }^{209} \mathrm{Bi}^{82+}$ & $9 / 2$ & 4.1106 & $5.2168 \times 10^{+00}$ & $-1.1042 \times 10^{-01}$ & $9.2617 \times 10^{-03}$ & $-6.1945 \times 10^{-02}$ & $5.0537 \times 10^{+00}$ & $2.4534 \times 10^{+02}$ \\
\hline${ }^{235} \mathrm{U}^{91+}$ & $7 / 2$ & -0.38 & $8.1170 \times 10^{-01}$ & $-2.4435 \times 10^{-02}$ & $1.6707 \times 10^{-03}$ & $-1.1226 \times 10^{-02}$ & $7.7771 \times 10^{-01}$ & $1.5942 \times 10^{+03}$ \\
\hline
\end{tabular}

Table 5. (Continued) 